hep-th/0703190

March 2007

\title{
Charting the Landscape of Modified Gravity
}

\author{
Nemanja Kaloper ${ }^{1}$ and Derrick Kiley ${ }^{2}$ \\ Department of Physics, University of California, Davis, CA 95616
}

\begin{abstract}
We explore brane induced gravity on a 3-brane in six locally flat dimensions. To regulate the short distance singularities in the brane core, we resolve the thin brane by a cylindrical 4-brane, with the geometry of $4 D$ Minkowski $\times$ a circle, which has an axion flux to cancel the vacuum pressure in the compact direction. We discover a large diversity of possible solutions controlled by the axion flux, as governed by its boundary conditions. Hence brane induced gravity models really give rise to a landscape of vacua, at least semiclassically. For sub-critical tensions, the crossover scale, below which gravity may look $4 D$, and the effective $4 D$ gravitational coupling are sensitive to vacuum energy. This shows how the vacuum energy problem manifests in brane induced gravity: instead of tuning the $4 D$ curvature, generically one must tune the crossover scale. On the other hand, in the near-critical limit, branes live inside very deep throats which efficiently compactify the angular dimension. In there, $4 D$ gravity first changes to $5 D$, and only later to $6 D$. The crossover scale saturates at the gravitational see-saw scale, independent of the tension. Using the fields of static loops on a wrapped brane, we check the perturbative description of long range gravity below the crossover scale. In sub-critical cases the scalars are strongly coupled already at the crossover scale even in the vacuum, because the brane bending is turned on by the axion flux. Near the critical limit, linearized perturbation theory remains under control below the crossover scale, and we find that linearized gravity around the vacuum looks like a scalar-tensor theory.
\end{abstract}

\footnotetext{
${ }^{1}$ kaloper@physics.ucdavis.edu

${ }^{2}$ dtkiley@physics.ucdavis.edu
} 


\section{Introduction}

\subsection{Prologue}

The mystery of the cosmological constant is arguably the most pressing and puzzling problem in contemporary fundamental physics. To date, the attempts to explain a small cosmological constant in the framework of effective field theory formulation of matter coupled to gravity, based on conventional lore of naturalness alone, have not yielded an answer (see the classic work [1] which still provides a state-of-the-art review). On the other hand, cosmological observations [2] strongly suggesting a small cosmological constant, and the discovery of the landscape of string vacua [3], have lent support to the idea of statistical selection of vacua, and the value of the cosmological constant in them [4]-[9], prompting a new debate about anthropic reasoning in physics.

One may try to alter the problem by embedding our universe in a fundamentally higherdimensional space-time in some way [10]-[12]. If, for example, our universe is a brane in extra dimensions, it would also have extrinsic curvature, and then one may divert the brane vacuum energy into the extrinsic curvature [12]. In this way, the vacuum energy could remain invisible to long distance $4 D$ gravity, yielding a very weakly curved universe. However, while attempting to recover $4 D$ General Relativity at large distances by compactifying the bulk, with covariantly conserved sources, one needed additional branes in the bulk, that restore a fine-tuning similar to the standard $4 D$ one $[12,13]$. The resulting picture was again that of a landscape: a theory admitting multiple $4 D$ vacua, classified by the free integration constants for some of the bulk fields (see, e.g.. [14]). Using these integration constants, one could hope to derive a framework where the effective cosmological constant could change in small amounts from one background to another. But one gains no more than that: the landscape reemerges, and one must retreat to either statistical or anthropic arguments to select a phenomenologically viable ground state.

A different idea for recovering $4 D$ General Relativity at large distances has been pursued in the so-called brane induced gravity theory [15]. In more interesting variants of braneinduced gravity, the extra-dimensional space has infinite volume. When the bulk volume is infinite, the $4 D$ graviton zero mode is not normalizable, and hence it completely decouples. Thus $4 D$ gravity ought to emerge from the exchange of the continuum of bulk modes, which are all massive from the $4 D$ viewpoint. To ensure this, [15] introduced induced curvature terms on the brane, arguing that they will be generated by brane quantum corrections anyway. If the scale $M_{4}$ that normalizes them is much greater than the bulk Planck scale, the brane kinetic terms would pull the bulk gravitons with wavelengths shorter than a certain crossover scale $r_{c}$ very close to the brane, yielding the momentum transfer due to the scattering of virtual bulk gravitons $\propto 1 / p^{2}$ for the momenta $p>r_{c}{ }^{-1}$, and a very small coupling of the three-point vertex $\sim 1 / M_{4}$. This gives a force which scales as $1 / r^{2}$, simulating $4 D$ behavior [15], and is nicely illustrated in the exact gravitational shock waves of [16].

This force contains admixtures of longitudinal, helicity-0 gravitons, and sometimes also radions. They couple gravitationally and their long range contributions to the force never completely disappears in perturbation theory [17]. However it has been argued, for massive gravity [18] and similarly for brane induced gravity [19], that the theory goes nonlinear 
at large distances from the source, and that the nonlinearities might screen away the extra scalar modes. On the other hand, this also indicates [20]-[23] that brane induced gravity runs into a strong coupling regime at macroscopic distances $r_{\text {strong }} \sim\left(r_{c}^{2} / M_{4}\right)^{1 / 3}$, requiring very low scale UV completions, and forcing the question of whether such completions even exist! The extra scalars may also turn into a perturbative ghost on self-accelerating backgrounds in $5 D$ bulks $[22,24,25,26,27,28]$. This suggests that the self-accelerating backgrounds are unstable, but the strong coupling at subhorizon scales obstruct the exploration of their fate in perturbation theory. Still, the shock waves $[16,25]$ can identify energy leaks from a self-accelerating brane into the bulk, that render the dynamics manifestly different from a $4 D$ one.

Similar mechanisms may also operate on higher-codimension defects [15, 29]. The authors of [29] have pursued ideas how UV regularizations of gravity might affect the crossover scale and the IR spectrum of the theory, hoping to suppress the extra graviton helicites. Subsequent discussions involved arguments as to how some regularizations may suffer from ghosts [30], which precisely cancel the extra graviton helicities, and how to avoid them $[31,32]$. The explicit constructions are hindered by the short-distance singularities in the core of the defect, which must be regulated before one can reliably calculate the low energy behavior of gravity.

We feel that exploring brane induced gravity on codimension-2 brane models is particularly interesting. In this case we can find the exact background solutions that play the role of the $4 D$ vacua directly, thanks to the magic of gravity in $3 D$. It is well known from early braneworld analysis that 3-branes in $6 D$ need not locally curve the bulk even if they carry tension [33]. Although this leads to flat $4 D$ geometries for nonvanishing tensions, it does not help with the cosmological constant problem after one compactifies the bulk to get long range $4 D$ gravity. This was immediately realized already in [33] to be in agreement with Weinberg's no-go theorem [1], and was discussed in more detail in [34]. Still, many interesting properties of gravity localized on codimension-2 defects, flat or not, in a $6 D$ bulk, compact and not, have been since explored in [35]-[52], including exact black holes and gravitational shock waves straddling the brane [50]. The sheer proliferation of such configurations points to a structure as rich as the landscape of supergravity solutions, albeit more exotic. Sketching a chart of this realm is the primary aim of the present paper, as we now elaborate.

\subsection{The Landscape of Brane Induced Gravity}

We begin our foray into the landscape of brane induced gravity by exploring thin static codimension-2 branes, which exist for sub-critical tensions $\lambda<\lambda_{c}=2 \pi M_{6}^{4}$ and have a flat $4 D$ induced metric. Because the brane induced curvature terms identically vanish, these solutions are identical to the ones in $6 D$ General Relativity with an empty bulk. We then construct exact gravitational shock waves of relativistic particles on the brane, generalizing the solutions of [50] to the framework of brane induced gravity. The shocks confirm that brane induced gravity on singular defects remains $4 D$ all the way to infinity $[15,29]$.

Yet, these solutions are pathological. Even an infinitesimal displacement of the probe from the brane into the bulk reveals that the thin brane theory is singular, since the gravitational field jumps from a finite value to exactly zero everywhere in the bulk [15, 29]. 
Mathematically, the discontinuities on thin branes can be attributed to an ill-posed exterior boundary value problem: modelling brane sources by points on an infinite cone introduces singularities both near and far. One turns it into a well-posed problem simply by replacing the point sources with finite rings, and considering the combined interior and exterior problems. Physically, this amounts to smearing the source over a finite space. We do it by replacing the thin 3-brane by a 4-brane wrapped on a circle, by adding an axionic field on the brane, whose vev breaks translations along the circle $[43,51]$. This is similar to the Scherk-Schwarz mechanism [53] for supersymmetry breaking and mass generation by dimensional reduction, an important ingredient of the string landscape model-building [3]. Much like in the string landscape, the axion 'charge' determines the radius of the compact circle $r_{0}$ for a given value of the tension, and we must choose it carefully to get the desired value of $r_{0}$. Alternatively, if some other dynamics were to fix $r_{0}$, we need to tune the axion charge to precisely cancel the pressure of the brane tension along the circle, because otherwise the background could not simulate the $4 D$ Minkowski vacuum from the get go.

The thick branes will still have a flat static geometry with an infinite bulk, but only for tensions smaller than the critical value. When the tension exceeds the critical value, our solutions show how the bulk compactifies and develops a naked singularity that soaks up the brane's gravitational field lines, just like the supercritical cosmic string in $4 D$ [54]. These solutions look like singular teardrop compactifications analyzed by Gell-Mann and Zwiebach in [55]. As with $4 D$ supercritical defects [54], one expects that for supercritical branes, as well as for the cases with a mismatch between the tension and the axion charge, there exist nonsingular solutions which describe some curved, nonstationary backgrounds. Examples which describe topologically inflating defects have been found recently in [56].

We next calculate the crossover scale where gravity changes from $4 D$, again using the shock wave solutions, sourced by relativistic strings moving on the wrapped brane. Below the crossover scale, the shock wave indeed approximates the $4 D$ Aichelburg-Sexl solution [57] down to $r_{0}$, whereas at much larger distances it changes over to the $6 D$ shock of [58], for sub-critical branes. The physics of crossover is very intricate: it is controlled by the deficit angle of the vacuum solution. This is because of the 'lightning rod' amplification of the bulk gravitational coupling, leading to $M_{6 \text { eff }}^{4}=(1-b) M_{6}^{4}[50]$, where $1-b=1-\frac{2 \lambda_{5} r_{0}}{M_{6}^{4}}$ measures the deficit angle of the brane. It is intuitively clear why it affects the gravitational force: on a cone, gravitational field lines spread more slowly than on a plane, and hence gravity must look stronger [50]. For sub-critical strings with deficit angle smaller than $2 \pi$, our exact crossover scale is qualitatively very different from the see-saw scale of [29], yielding instead $r_{c}^{2} \sim \frac{M_{4}^{2}}{M_{6 \text { eff }}^{4}}$, up to a $\log$, where $M_{4}$ is the effective $4 D$ Planck scale found by compactifying the wrapped 4-brane theory on the circle of radius $r_{0}$.

On the other hand, in the near-critical limit the bulk compactifies to a cylinder, which opens up into a cone only very far from the brane, at distances $\gtrsim \frac{r_{0}}{1-b}$. In the cylindrical throat gravity is in a $5 D$ gravity regime, separating the $4 D$ and $6 D$ ones. Thus the crossover scale beyond which gravity is not $4 D$ is really the demarcation between the $4 D$ regime and $5 D$ gravity which lives inside the throat [59]. To see where this happens, we can use the naive crossover formula on a codimension-1 brane, realized as a wrapped 4-brane in a $6 D$ flat space with a compact circle: by Gauss formulas for Planck masses $M_{4 e f f}^{2}=M_{5}^{3} r_{0}$ and 
$M_{5 \text { eff }}^{3}=M_{6}^{4} r_{0}$, we find exactly $r_{c} \sim \frac{M_{4 \text { eff }}^{2}}{M_{5 \text { eff }}^{3}} \sim \frac{M_{5}^{3}}{M_{6}^{4}}$, which is our crossover formula from the exact shock waves, and which is the same as the see-saw scale $r_{c} \sim \frac{M_{4}^{2}}{M_{6}^{4} r_{0}}$ of [29]. Thus we see precisely how the see-saw mechanism emerges.

Our analysis reveals the presence of a large topographic diversity of solutions. The axion flux which cancels the pressure in the compact direction sets the radius of the circle by $q^{2}=2 \lambda_{5} r_{0}^{2}$. Since the $4 D$ Planck scale is $M_{4}^{2} \sim M_{5}^{3} r_{0} \sim \frac{M_{5}^{3} q}{\sqrt{\lambda_{5}}}$, and, for sub-critical branes, the crossover scale $r_{c} \sim \frac{M_{5}^{3} q}{\sqrt{2 \lambda_{5}} M_{6}^{4}-2 \lambda_{5} q}$ we can find the charge $q$ that will produce any desired $M_{4}$ for any values of $M_{5}$ and $\lambda_{5}$. But then, the crossover scale is uniquely fixed in terms of these parameters. The vacuum energy problem reappears in brane induced gravity: although the brane is flat, when we fix $M_{4}$, we must finely tune both the axion charge and the tension of the brane to get $r_{c}$ to take some desired value on a sub-critical brane. On the other hand, once we fix $\lambda_{5}, M_{5}$ and $M_{6}$, and let the charge $q$ vary, we will find that the thickness, the effective $4 D$ Planck scale, and, for sub-critical branes, the crossover scale of the brane also vary, spanning a wide range of theories below the crossover scale. The near-critical branes, where the deficit angle approaches $2 \pi$, are more interesting since their crossover scale saturates at $r_{c} \sim \frac{M_{5}^{3}}{M_{6}^{4}}$, which is completely independent of the brane tension in the leading order. This is because their conical throat [59] shields them from the asymptotic infinity. Only their effective $4 D$ Planck mass is sensitive to the brane tension, and changes when the tension varies and the axion charge is fixed. The charge and the tension still need to be tuned right to get a desired value of $M_{4}$, but this may be substantially easier.

This is a signature of a landscape: low energy parameters of a theory depend on boundary conditions, instead of being uniquely fixed by a symmetry principle. We could also imagine a brane where $q$ and $\lambda_{5}$ might slowly vary along the brane, leading to regions of $4 D$ space with a different strength of simulated $4 D$ gravity, but always with a flat static $4 D$ vacuum for sub-critical vacuum energies! Thus, we unveil a classical landscape of codimension-2 brane induced gravity, where the brane may remain flat since the curvature is soaked up by the deficit angle, but $M_{4}$ and generically $r_{c}$ depend on the vacuum energy.

Including quantum mechanics in this picture requires a lot of care. An immediate, semiclassical issue is that since the axion is a phase of some complex $5 D$ scalar field, its charge is quantized in the units of a new UV mass scale $\mu$. The quantization law, $q=\mu^{3 / 2} n$, where $n$ is an integer winding number, yields $M_{4}^{2} \sim \frac{M_{5}^{3} \mu^{3 / 2}}{\sqrt{\lambda_{5}}} n$, so that for a given set of dimensional parameters we must choose the right winding number. The value of $M_{4}$ changes discretely with it. Similarly, for sub-critical branes, the crossover scale can only change in discrete jumps on static backgrounds. Thus the static configurations will only exist at discrete locations of the landscape, most of which is covered by nonstationary solutions, like in the string landscape with a discretuum of vacua [3]. Finding the nonstationary solutions is outside of the scope of this work; we hope to return to it elsewhere. Nevertheless as long as gravity is treated classically and field theory is consistently cut off at some scale in the UV, this can be done in principle, showing that the classical landscape which we glimpse at can be extended semiclassically. However, there remains the looming question about UV completions of brane induced gravity models, and their embeddings into the theories of quantum gravity $[15,60,61,62]$. We will have nothing new on this to add here, taking a more pedes- 
trian approach of simply charting out the (semi)classical countenance of what the quantum landscape might be.

The shock wave solutions have taught us that at distances between $r_{0}$ and $r_{c}$, the theory contains $4 D$ General Relativity. To see what else is there, we develop, in painstaking detail, linearized perturbation theory about a wrapped brane. For sub-critical tension the scalars are strongly coupled essentially exactly at the crossover scale $r_{c}$, which is the Vainshtein scale [18] of the vacuum itself. This happens because the scalar gravitons probe the asymmetric vacuum energy distribution, set up by the background axion flux, which triggers brane bending. However in the near-critical limit linearized perturbation theory around the vacuum remains under control below the crossover scale. Solving for the linearized gravitational field of a static uniform ring of mass on the brane, we find that below the crossover scale the theory contains helicity-0 and radion-like scalars in addition to the helicity- 2 modes. The radion modes decouple but the helicity-0 modes remain active at the linearized level. Thus the linearized theory around near-critical vacua approximates a Brans-Dicke gravity with $\omega=0$. This would disagree with the classic tests of General Relativity [17], but perhaps non-linearities or dynamics on vacua of more complex structure could come to the rescue. We however find that rings of brane matter built of the lightest KK states on the wrapped brane do not entice any fast instabilities in the leading order of perturbation theory. While we suspect that fast instabilities might remain absent in perturbation theory beyond linear order, we have not proven it, and it remains a question for future studies.

The paper is organized as follows: we begin with a lightning review of thin codimension-

2 flat branes and their short distance singularities, as revealed by shock waves, in section 2. We then construct the regulated backgrounds, with a 4-brane wrapped on a circle, in section 3. We also discuss how critical and supercritical branes compactify the bulk, the latter inducing a naked singularity far away. We derive regulated shock wave solutions in section 4, to explore the long range properties of gravity and compute the crossover scale. In section 5 we set up linear perturbation theory, and give the linearized solution of a static ring of mass on the 4-brane. We end with conclusions.

\section{Tensional Thin Branes on Bulk Cones}

\section{$2.1 \quad$ Vacua}

The field equations describing a 3-brane in an empty $6 D$ bulk in brane induced gravity are $[15,29]$, in a brane fixed Gaussian-normal gauge,

$$
M_{6}^{4} G_{6}{ }_{B}^{A}+M_{4}^{2} G_{4}{ }_{\nu}{ }_{\nu} \delta^{A}{ }_{\mu} \delta^{\nu}{ }_{B} \delta^{(2)}(\vec{y})=T^{\mu}{ }_{\nu} \delta^{A}{ }_{\mu} \delta^{\nu}{ }_{B} \delta^{(2)}(\vec{y})
$$

Our conventions are that the indices $A, B, \ldots$ count all spacetime dimensions, while $\mu, \nu, \ldots$ run over the brane worldvolume. The $\delta$-function is the tensor $\delta^{(2)}(\vec{y})=\frac{\sqrt{g_{4}}}{\sqrt{g_{6}}} \Pi \delta\left(y_{i}\right)$, given by the normalization of the usual tensor density by the metric determinant of the extra space, coordinatized by $\left(y_{1}, y_{2}\right)$ (we will use $x^{\mu}$ for the coordinates along the brane worldvolume). As in $[15,29]$ we take the stress-energy tensor $T^{\mu}{ }_{\nu}$ to be localized on the brane, separating the tension $\lambda$ from the matter sources by $T^{\mu}{ }_{\nu}=-\lambda \delta^{\mu}{ }_{\nu}+\tau^{\mu}{ }_{\nu}$. 
The vacua of this theory are maximally symmetric solutions with $\tau_{\nu}^{\mu}=0$. Substituting this in (1), imposing a flat brane $g_{\mu \nu}=\eta_{\mu \nu}$ and tracing over, we find $R_{2}=\frac{2 \lambda}{M_{6}^{4}} \delta^{(2)}(\vec{y})$, where $R_{2}$ is the curvature in the spatial dimensions transverse to the brane. The solution is a conical space [33], which can be seen most simply as follow. Since any metric in two dimensions is conformally flat, we can write $d s_{2}{ }^{2}=e^{-\vartheta} d \vec{y}^{2}$. Then by conformal flatness of the metric, $R_{2}=e^{\vartheta} \vec{\nabla}_{y}^{2} \vartheta$, and recalling the Euclidean $2 D$ Green's function $\ln (|\vec{y}| / \ell)$, which obeys $\vec{\nabla}_{y}^{2} \ln (|\vec{y}| / \ell)=2 \pi \Pi \delta\left(y_{i}\right)$, we see that to get the solution we can set $\vartheta=2 b \ln (|\vec{y}| / \ell)$ to find $R_{2}=4 \pi b e^{2 b \ln (|\vec{y}| / \ell)} \Pi \delta\left(y_{i}\right)$. We next compare this to $R_{2}=\frac{2 \lambda}{M_{6}^{4}} \delta^{(2)}(\vec{y})=\frac{2 \lambda}{M_{6}^{4}} e^{2 b \ln (|\vec{y}| / \ell)} \Pi \delta\left(y_{i}\right)$ from above, from which we finally obtain

$$
b=\frac{\lambda}{2 \pi M_{6}^{4}} .
$$

The arbitrary length scale $\ell$, needed for dimensional reasons, completely drops out of these equations. Then substituting the solution for $\vartheta$, going to spherical polar coordinates and changing the radial coordinate to $\rho=\frac{1}{1-b} b^{b}|\vec{y}|^{1-b}$, we get

$$
d s_{2}{ }^{2}=d \rho^{2}+(1-b)^{2} \rho^{2} d \phi^{2} .
$$

Since the range of the angular variable $\phi$ is $2 \pi, 1-b$ is the deficit angle induced by the tension, as per Eq. (2). The full $6 D$ vacuum solution is

$$
d s_{6}^{2}=\eta_{\mu \nu} d x^{\mu} d x^{\nu}+d \rho^{2}+(1-b)^{2} \rho^{2} d \phi^{2} .
$$

This is identical to the thin 3-brane in a flat bulk found in models with large extra dimensions [33] because the induced curvature identically vanishes on flat branes. The bulk geometry is a cone, given in Fig. (1). It is well known, however, that for backgrounds with matter sources on a thin brane in higher-dimensional gravity the short distance structure of the geometry near the brane is more involved [46]-[48, 50, 51]. In general, the length scale $\ell$ does not drop out but needs to be promoted into a UV regulator, smoothing the tip of the cone in Fig. (1). As we will see below this becomes even more important in brane induced gravity.

Clearly, we see that the solution (4) is not well defined at $b=1$, corresponding to the critical value of the tension $\lambda_{c r}=2 \pi M_{6}^{4}$. While the metric (4) makes sense, as it stands, for $b>1$, it is not clear what it represents since we can't smoothly deform it to relate it to sub-critical cases. Hence in the critical limit, and beyond it, we cannot tell what happens to the brane until we sort out how to match the brane's core to the exterior, in precisely the same manner as for the critical and super-critical strings [54].

\subsection{Shocks on Thin Branes}

A simple way to probe the nature of gravity, as argued in $[16,50]$, once a vacuum solution is known, is to shock it. By this we mean, put a relativistic particle on a background, use its stress energy tensor as a source, and solve the equations for its gravitational field. Because of Lorentz boosts, the field becomes completely confined to the space transverse to the direction 


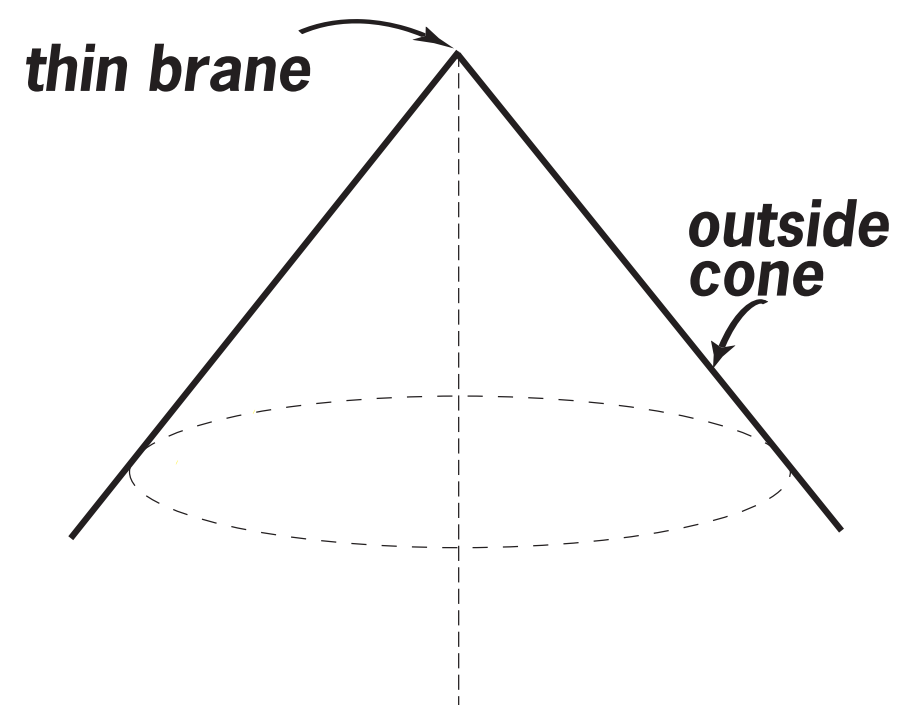

Figure 1: $2 D$ conical bulk geometry of a thin brane vacuum.

of motion, and hence the field equations, however formidable they may be, will linearize. A straightforward technique to solve them is to use the cutting and pasting technique of Dray and 't Hooft $[65,66]$, which works in our case since the sub-critical vacuum metric Eq. (4), with $b<1$, is locally flat. So: the shocked metric will be

$$
d s_{6}{ }^{2}=4 d u d v-4 \delta(u) f\left(\vec{x}_{\perp}, \vec{y}\right) d u^{2}+d \vec{x}_{\perp}^{2}+d \rho^{2}+(1-b)^{2} \rho^{2} d \phi^{2},
$$

where $\vec{x}_{\perp}$ coordinatize the two directions along the brane transverse to the particle that moves along the null geodesic $u=0$, and $\vec{y}$ are the coordinates on the cone. The function $f$ is the shock wave profile that we need to solve for. Then, using Eq. (5) as an ansatz, we substitute it into the field equations Eq. (1), with the relativistic stress energy tensor $\tau_{\nu}^{\mu}=\frac{2 p}{\sqrt{g_{4}}} g_{4 u v} \delta(u) \delta^{(2)}\left(\vec{x}_{\perp}\right) \delta^{\mu}{ }_{v} \delta^{u}{ }_{\nu}$ of the source with 4-momentum $p^{\mu}=(p, 0,0, p)$ included, and determine the equation controlling the shock wave profile $f$.

Since for relativistic particles $\tau_{\mu}^{\mu}=0$, we still have $R_{6}=\frac{2 \lambda}{M_{6}^{4}} \delta^{(2)}(\vec{y})$ and $R_{4}=0$ regardless of the source. We substitute this in the field equations along the brane worldvolume,

$$
M_{6}^{4} R_{6}{ }_{\nu}{ }_{\nu}+M_{4}^{2} R_{4}{ }^{\mu}{ }_{\nu} \delta^{(2)}(\vec{y})=\tau^{\mu}{ }_{\nu} \delta^{(2)}(\vec{y}) .
$$

The field equations in the bulk, $R_{6}=\frac{2 \lambda}{M_{6}^{4}} \delta^{(2)}(\vec{y})$, are trivially solved by Eq. (5) and so all the new information is completely contained in (6). The solution of these equations will be consistent, since one can easily verify that $\nabla_{\mu} \tau^{\mu}{ }_{\nu}=0$. Now, the only nontrivial components of the Ricci tensors for the metric (5) are

$$
\begin{aligned}
& R_{6}{ }^{v}{ }_{u}=\delta(u) \nabla_{4}{ }^{2} f, \\
& R_{4}{ }^{v}{ }_{u}=\delta(u) \nabla_{2}{ }^{2} f,
\end{aligned}
$$


where $\nabla_{4}^{2}$ is the full transverse Laplacian, and $\nabla_{2}^{2}$ its restriction on the $2 D$ transverse plane on the brane, coordinatized by $\vec{x}_{\perp}$. Substituting this and the formula for $\tau^{\mu}{ }_{\nu}$ in Eq. (6) we finally obtain the field equation for $f$ :

$$
\nabla_{4}^{2} f+\frac{M_{4}^{2}}{M_{6}^{4}} \nabla_{2}^{2} f \delta^{(2)}(\vec{y})=\frac{2 p}{M_{6}^{4}} \delta^{(2)}\left(\vec{x}_{\perp}\right) \delta^{(2)}(\vec{y}) .
$$

All other equations in (6) are trivially satisfied on (5). Thus we see that the problem of determining the configuration which solves (modified) gravity equations for relativistic sources, as before, maps onto a much simpler problem of solving a (modified) Poisson equation for a static charge [65]-[66],[16]. This illustrates the power of shock therapy as a diagnostic method. Once we have the solution of Eq. (8), we can compare it to the Aichelburg-Sexl solution [57] to see if, and how, the theory mimics $4 D$ General Relativity.

Let us now solve Eq. (8), at least formally. Fourier transforming in the brane transverse space

$$
\delta^{2}\left(\vec{x}_{\perp}\right)=\frac{1}{(2 \pi)^{2}} \int d^{2} \vec{k} e^{i \vec{k} \cdot \vec{x}_{\perp}}, \quad f=\frac{1}{(2 \pi)^{2}} \int d^{2} \vec{k} \varphi_{k}(\vec{y}) e^{i \vec{k} \cdot \vec{x}_{\perp}}
$$

using $\nabla_{4}^{2}=\nabla_{\vec{y}}^{2}+\nabla_{2}^{2}$, where the operator $\nabla_{\vec{y}}^{2}$ is the Laplacian on the $2 D$ cone, and replacing $\delta^{(2)}(\vec{y})=\frac{1}{2 \pi(1-b) \rho} \delta(\rho)$ by using the axial symmetry of (5) and the null source $\tau^{\mu}{ }_{\nu}$, after a little algebra we obtain

$$
\left(\nabla_{\vec{y}}^{2}-k^{2}\right) \varphi_{k}=\frac{1}{2 \pi(1-b) M_{6}^{4}}\left(2 p+M_{4}^{2} k^{2} \varphi_{k}\right) \frac{\delta(\rho)}{\rho}
$$

which is very similar to the field equation of a bulk scalar with kinetic terms induced on the brane [15]. Note that the bulk coupling $M_{6}^{-4}$ has been replaced by the effective coupling $\left[(1-b) M_{6}^{4}\right]^{-1}$ which is stronger on the cone because of its deficit angle, representing the 'lightning rod' amplification effect pointed out in [50]. At any rate, we can now use the same tricks to solve it as [15]. Ignoring the angular variable by axial symmetry, and substituting $\varphi_{k}(\rho)=D(k, \rho) F(k)$ where $D$ obeys the Helmholtz equation

$$
\left(\nabla_{\vec{y}}^{2}-k^{2}\right) D=\frac{2 p}{2 \pi(1-b) M_{6}^{4}} \frac{\delta(\rho)}{\rho},
$$

we then determine $F$ such that the ansatz $\varphi_{k}=D F$ correctly solves Eq. (9). This yields $F=\left(1-\frac{M_{4}^{2}}{2 p} k^{2} D(k, 0)\right)^{-1}$, and therefore the momentum space field $\varphi_{k}$ is

$$
\varphi_{k}=\frac{D(k, \rho)}{1-\frac{M_{4}^{2}}{2 p} k^{2} D(k, 0)} .
$$

The Helmholtz equation is defined on the full $2 D$ cone, with the source residing exactly on its vertex, and so we can write its solution $D$ in terms of the modified Bessel function $K_{0}$, which vanishes at infinity and hence describes properly gravity localized to the brane as

$$
D(k, \rho)=-\frac{p}{\pi(1-b) M_{6}^{4}} K_{0}(k \rho) .
$$


where we have explicitly used the fact that the other modified Bessel function $I_{0}$ is equal to unity on the brane at $\rho=0$, while it diverges at infinity. We point it out since it will arise naturally later on, although here it plays no role yet. Then, substituting this in $\varphi_{k}$ and Fourier transforming it back to the transverse configuration space along the brane, and integrating over the angular bulk variable in the usual way ${ }^{1}$ we finally find the solution for the shock wave profile on a thin 3-brane:

$$
f=-\frac{p}{2 \pi^{2}(1-b) M_{6}^{4}} \int_{0}^{\infty} d k \frac{k K_{0}(k \rho) J_{0}\left(k\left|\vec{x}_{\perp}\right|\right)}{1+\frac{M_{4}^{2}}{\pi(1-b) M_{6}^{4}} k^{2} K_{0}(0)} .
$$

This solution is very interesting - because it is pathological. Its pathologies stem from the fact that $K_{0}$ diverges in the core of the brane. Indeed, $K_{0}(\epsilon) \sim-\ln (\epsilon) \rightarrow \infty$ as $\epsilon \rightarrow 0$. However, along the brane, at $\rho=0$, the divergence precisely cancels between the numerator and the denominator in Eq. (14). In this case, we find

$$
f=-\frac{p}{2 \pi M_{4}^{2}} \int_{0}^{\infty} d k \frac{J_{0}\left(k\left|\vec{x}_{\perp}\right|\right)}{k}=\frac{p}{\pi M_{4}^{2}} \ln \left(\frac{\left|\vec{x}_{\perp}\right|}{\ell}\right)
$$

which is exactly the $4 D$ Aichelburg-Sexl solution - at all distances along the 3-brane! This result would be incredibly interesting, because it occurs despite the infinite bulk space surrounding it, that could be explored by the graviton multiplet. This looks like a very efficient mechanism to hide extra dimensions from the gravitational exploration. However, if we move off the brane by an even infinitesimal amount, to $\rho=\epsilon \neq 0$, from (14) we find that the divergence of the denominator forces the shock wave profile immediately to zero. Thus the confinement of gravity to the brane is perfect - but it happens because the Bessel function diverges in the core, and is clearly a UV sensitive answer, as indeed noted in [15, 29]. If we consider a physically more realistic brane of finite thickness, the confinement may be far less than perfect, leading to the reopening of extra dimensions to gravity at some finite distance from the source [29]. Therefore, before we draw any conclusions about brane induced gravity in codimension $\geq 2$, we first must regulate the short distance singularities inside the brane.

Our shock wave example points us to a very simple way how to regulate. The discontinuity of the solution is due to the divergence in the function $K_{0}(\rho)$ at $\rho=0$. Mathematically, the divergence of $K_{0}$ on the source is the familiar pathology from an ill-posed problem for an elliptic Helmholtz equation for a pointlike source, which does not have a regular exterior solution in $2 D$. A well known prescription for regulating this problem is to replace the pointlike source by a ring-like one. We can then split the problem into a combination of interior and exterior problems, which have well behaved solutions for ring sources of a finite size. Since our pointlike source is really a particle on a codimension- 2 brane, a natural way to replace it by a ring-like source is to consider a loop of string moving on a cylinder. If we then want to derive the background cylinder from a covariant action, the easiest way to do it is to take a 4-brane and wrap it around a circle of finite size. Such tricks were used earlier in $[43,51]$ and we employ it next.

\footnotetext{
${ }^{1}$ We use $f=\frac{1}{(2 \pi)^{2}} \int d^{2} \vec{k} \varphi_{k} e^{i \vec{k} \cdot \vec{x}_{\perp}}$ and integrate over $\phi$ to get $f=\frac{1}{2 \pi} \int_{0}^{\infty} d k k \varphi_{k} J_{0}\left(k\left|\vec{x}_{\perp}\right|\right)$, as in any cylindrically symmetric problem in potential theory.
} 


\section{Wrapped Branes and Truncated Cones}

\subsection{Making Vacua}

We wish to replace the thin brane vacuum (4) by one where the brane has a core of finite thickness, but its metric remains the same as (4) outside of the brane core. In this case the thick brane vacuum will retain some of the attractive features of the thin 3-brane, namely $4 D$ flatness and the presence of $4 D$ General Relativity, for the sub-critical case. Furthermore, we will find a simple model which will enable us to see what goes on as the tension approaches and exceeds the critical limit. Now, the reason why the metric Eq. (4) solved the field equations (1) was that the stress energy tensor of a tensional 3-brane has the form $T^{\mu}{ }_{\nu}=-\lambda \delta^{(2)}(\vec{y})$ along the brane and vanished away from it. In component notation, this looks like $T_{B}^{A}=-\lambda \delta^{(2)}(\vec{y}) \operatorname{diag}(1,1,1,1,0,0)$. A very simple model of a 3-brane with a finite core is a cylinder: a 4-brane wrapped on a circle $[43,51]$. However, when the 4-brane has nonzero tension, with the vacuum action $S_{\text {vacuum }}=-\int d^{5} x \sqrt{g_{5}} \lambda_{5}$, its stress energy tensor will be $T_{B}^{A}=-\lambda_{5} \delta\left(\rho-r_{0}\right) \operatorname{diag}(1,1,1,1,1,0)$, where we wound it around the circle of radius $r_{0}$, with $\rho$ along the normal to the brane. Thus, to wrap the 4-brane into a cylinder, we must cancel the pressure $\propto \lambda_{5}$ in the compact direction.

A simple way to do it is to put an axion-like field $\Sigma$ on the 4 -brane, with a vacuum action

$$
S_{\text {vacuum }}=-\int d^{5} x \sqrt{g_{5}}\left(\lambda_{5}+\frac{1}{2} g^{a b} \partial_{a} \Sigma \partial_{b} \Sigma\right),
$$

and look for solutions where $\Sigma$ breaks the translational invariance in the compact direction, but so that its stress energy tensor remains translationally symmetric. This is precisely the method of Scherk and Schwarz [53] for dimensionally reducing supergravities to get massive lower dimensional theories. The stress energy tensor obtained by varying Eq. (16) along the 4-brane is

$$
T^{a}{ }_{b}=-\lambda_{5} \delta^{a}{ }_{b}+\partial^{a} \Sigma \partial_{b} \Sigma-\frac{1}{2} \delta^{a}{ }_{b} g^{c d} \partial_{c} \Sigma \partial_{d} \Sigma,
$$

where lower case latin indices $a, b, \ldots$ run over 4 -brane worldvolume values $0,1, \ldots, 4$. Taking the fourth coordinate to be the angle on the compact circle $\phi$ and substituting the ScherkSchwarz ansatz $\Sigma=q \phi$ we choose $q$ to precisely cancel $T_{\phi}^{\phi}$. This requires

$$
\lambda_{5}=\frac{1}{2} q^{2} g^{\phi \phi},
$$

where $g^{\phi \phi}=\frac{1}{r_{0}^{2}}$ is the inverse radius squared of the compact dimensions. Then the remaining components of the 4-brane stress energy become precisely $T^{\mu}{ }_{\nu}=-2 \lambda_{5} \delta^{\mu}{ }_{\nu}$. Thus the brane source now reads $T_{B}^{A}=-2 \lambda_{5} \delta\left(\rho-r_{0}\right) \operatorname{diag}(1,1,1,1,0,0)$. The tensor structure is precisely the same as in the stress energy tensor of a thin 3 -brane. Now, since the thin brane is axially symmetric from the bulk viewpoint, we can rewrite the scalar $\delta$-function in polar coordinates as $\delta^{(2)}(\vec{y})=\frac{1}{2 \pi \rho} \delta(\rho)$. By inspection, we see that if we shift the argument of the remaining radial $\delta$-function to $\rho-r_{0}$, it becomes $\delta_{\text {thick }}^{(2)}(\vec{y})=\frac{1}{2 \pi r_{0}} \delta\left(\rho-r_{0}\right)$, and so to model the thick brane by a wrapped 4 -brane, we need to identify $\lambda \delta_{\text {thick }}^{(2)}(\vec{y})$ with $2 \lambda_{5} \delta\left(\rho-r_{0}\right)$. This yields

$$
\lambda=4 \pi r_{0} \lambda_{5},
$$


relating the $5 D$ and the effective $4 D$ vacuum energies. The effective $4 D$ vacuum energy $\lambda$ contains the contributions from the classical axion field $\Sigma$, doubling up its value, because of the cancellation condition of Eq. (18). This is equivalent to the effective vacuum energy contributions in the string landscape [3], which is also comprised of the contributions from quantum fields (here, $\lambda_{5}$ ) and classical fluxes (here $\propto q^{2}$ ). In the landscape, these contributions need to be finely adjusted to yield a $4 D$ Minkowski vacuum. Here, the situation is similar: because this stress energy is completely localized on a codimension- 1 cylindrical surface of finite radius, one expects that the solution representing this configuration should be obtained from patching locally flat $6 D$ Minkowski spaces inside and outside of the cylinder. The exterior should have a deficit angle by the construction of the thick brane stress energy, which shows that our 4-brane source looks precisely like the thick 3-brane.

Indeed, the field equations for a 4 -brane in a $6 D$ bulk which include brane localized gravity terms are $[15,29]$, in Gaussian-normal gauge, and with the 4-brane residing at $\rho=r_{0}$,

$$
M_{6}^{4} G_{6}{ }^{A}{ }_{B}+M_{5}^{3} G_{5}{ }_{b} \delta^{A}{ }_{a} \delta^{b}{ }_{B} \delta\left(\rho-r_{0}\right)=T^{a}{ }_{b} \delta^{A}{ }_{a} \delta^{b}{ }_{B} \delta\left(\rho-r_{0}\right),
$$

which we apply to our cylindrical brane vacuum, imposing that the stress energy tensor is covariantly conserved, that requires $\partial^{a} \partial_{a} \Sigma=0$. It is easy to check that by axial symmetry the ansatz $\Sigma=q \phi$ trivially solves the latter equation. Then, the tensor structure of the source, $T^{A}{ }_{B}=-2 \lambda_{5} \delta\left(\rho-r_{0}\right) \operatorname{diag}(1,1,1,1,0,0)$, guarantees that the flat $4 D$ metric is a solution, if the tension is again off-loaded into the bulk, like in the thin 3-brane case. Tracing the field equations (20), and using (18) we find that the condition for this is that the metric in the remaining two dimensions, coordinatized by the angular direction along the brane and the radial distance away from it has curvature

$$
R_{2}=\frac{4 \lambda_{5}}{M_{6}^{4}} \delta\left(\rho-r_{0}\right)
$$

Thus, by the arguments identical to those we invoked in the construction of the thin 3brane solutions, we can choose the coordinates where the $2 D$ metric is $d s_{2}^{2}=e^{-\vartheta} d \vec{z}^{2}$, where however the brane now resides at $r_{0}=\left|\vec{z}_{0}\right|$ around the origin. Substituting in (21), we find $R_{2}=e^{-\vartheta} \vec{\nabla}_{2}^{2} \vartheta$. Comparing to (21) and using the fact that $\vec{\nabla}_{2}^{2}\left[\Theta\left(r-r_{0}\right) \ln \left(r / r_{0}\right)\right]=\delta\left(r-r_{0}\right) / r$, we infer that

$$
\vartheta=2 b \Theta\left(r-r_{0}\right) \ln \left(\frac{r}{r_{0}}\right)
$$

where $\Theta(x)$ is the Heaviside step function, $\Theta(x)=0$ for $x<0$ and $\Theta(x)=1$ for $x \geq 0$, and $b$ is given precisely by combining Eqs. (2) and (19),

$$
b=\frac{2 \lambda_{5} r_{0}}{M_{6}^{4}} .
$$

The solution is given by $d s_{6}{ }^{2}=\eta_{\mu \nu} d x^{\mu} d x^{\nu}+e^{-2 b \Theta\left(r-r_{0}\right) \ln \left(r / r_{0}\right)}\left(d r^{2}+r^{2} d \phi^{2}\right)$, in polar coordinates. After defining the new radial coordinate $\rho$ by $d \rho / d r=\left(r_{0} / r\right)^{b \Theta\left(r-r_{0}\right)}$, such that

$$
\rho=\frac{r}{1-b \Theta\left(r-r_{0}\right)}\left(\frac{r_{0}}{r}\right)^{b \Theta\left(r-r_{0}\right)}-\frac{b r_{0} \Theta\left(r-r_{0}\right)}{1-b \Theta\left(r-r_{0}\right)},
$$


and $\Theta\left(r-r_{0}\right)=\Theta\left(\rho-r_{0}\right)$, we can rewrite the metric as

$$
d s_{6}{ }^{2}=\eta_{\mu \nu} d x^{\mu} d x^{\nu}+d \rho^{2}+\left[\left(1-b \Theta\left(\rho-r_{0}\right)\right) \rho+b r_{0} \Theta\left(\rho-r_{0}\right)\right]^{2} d \phi^{2} .
$$

For $\rho<r_{0}$ the $2 D$ part of the metric is $d s_{2}{ }^{2}=d \rho^{2}+\rho^{2} d \phi^{2}$, i.e. a flat disk, while for $\rho>r_{0}$ it becomes $d s_{2}{ }^{2}=d \rho^{2}+\left((1-b) \rho+b r_{0}\right)^{2} d \phi^{2}=d \rho^{2}+(1-b)^{2}\left(\rho+\frac{b}{1-b} r_{0}\right)^{2} d \phi^{2}$, precisely the metric on a cone, which we see by comparing it to the thin 3-brane metric from the previous section. Thus the combined solution represents a truncated cone, with a flat mesa of radius $r_{0}$ on top, as depicted in Fig. (2). The geometry of the brane is $4 D$ Minkowski $\times$ circle.

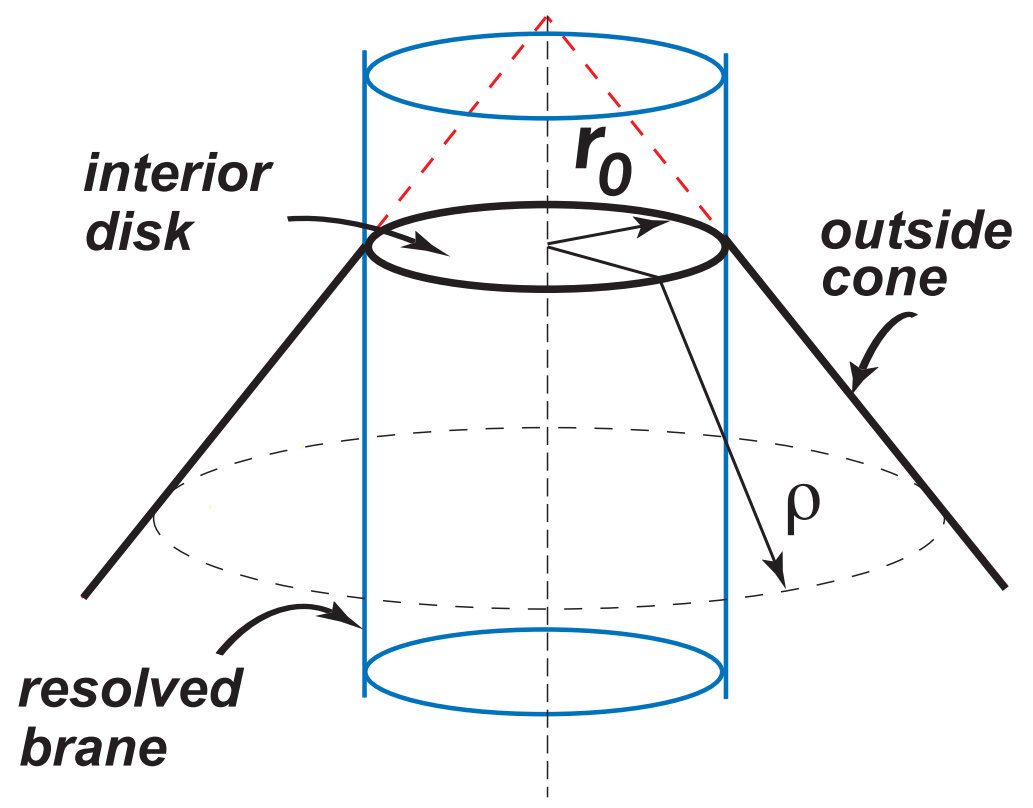

Figure 2: $2 D$ bulk geometry of the resolved brane.

Looking at Eq. (25) for $\rho>r_{0}$ we see that the critical string tension, where the deficit angle becomes $2 \pi$, is again given by $b=1$, which now corresponds to the tension

$$
\lambda_{5 c r}=\frac{M_{6}^{4}}{2 r_{0}} .
$$

Comparing to the relation between the $5 D$ tension $\lambda_{5}$ and the effective $4 D$ tension $\lambda$, given by Eq. (19) we find that the critical value of the effective $4 D$ tension is, not surprisingly, $\lambda_{c r}=2 \pi M_{6}^{4}$. We can now see what happens as $\lambda \rightarrow \lambda_{c r}$ and beyond. Since we are holding the brane core fixed, using its own internal dynamics, all we need to do is analyze the region $\rho>r_{0}$ of Eq. (25). When $b=1$, the metric outside of the brane changes to

$$
d s_{6}{ }^{2}=\eta_{\mu \nu} d x^{\mu} d x^{\nu}+d \rho^{2}+r_{0}^{2} d \phi^{2},
$$

which describes a cylinder $M_{4} \times R \times S^{1}$, where the radius of $S^{1}$ is the same as the brane radius. Now, when $b=1-\epsilon$, it's easy to see that the bulk cone looks like a sliver. In this 
limit, the exterior bulk metric is approximately $d s_{6}{ }^{2}=\eta_{\mu \nu} d x^{\mu} d x^{\nu}+d \rho^{2}+\left(\epsilon\left(\rho-r_{0}\right)+r_{0}\right)^{2} d \phi^{2}$. This looks like the metric on a cylinder for $r_{0} \leq \rho \lesssim r_{0} / \epsilon$, because the variation of the radius of the sliver, due to the change of radial distance $\rho$, is very small compared to the radius of the brane $r_{0}$. Near-critical branes reside inside deep bulk throats that only asymptotically open into conical geometries [59], see Fig (3).

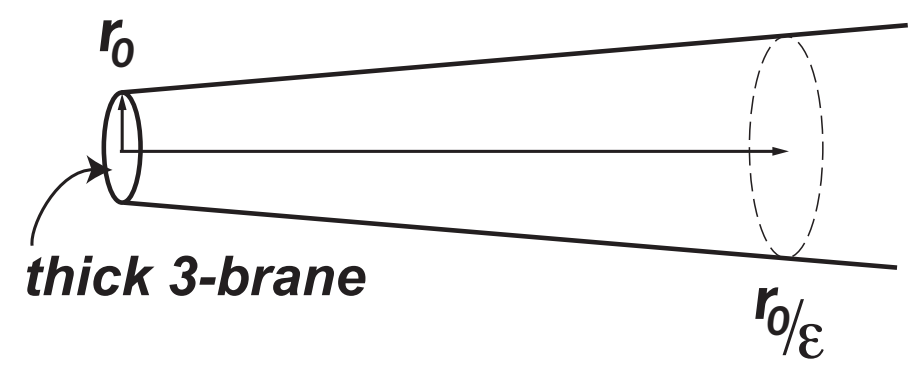

Figure 3: $2 D$ bulk geometry of the near-critical resolved 3-brane.

For supercritical branes, $b>1$, the exterior metric is just

$$
d s_{6}{ }^{2}=\eta_{\mu \nu} d x^{\mu} d x^{\nu}+d \rho^{2}+(b-1)^{2}\left(\rho-\frac{b}{b-1} r_{0}\right)^{2} d \phi^{2},
$$

which describes a bulk geometry that near $\rho=r_{0}$ looks like a flat disk. However now the metric has a singularity at $\rho=\frac{b}{b-1} r_{0}>r_{0}$, where the geometry tapers off to a conical spike. Thus when $b>1$ the bulk spontaneously compactifies on a $2 D$ teardrop, precisely of the shape discussed by [55]. Hence static flat branes in an infinite bulk will only occur for sub-critical values of the tension. In light of the discussions of [54], one expects that the supercritical brane solutions without bulk singularities exist, but that they describe nonstationary backgrounds. Examples describing topologically inflating domain walls on codimension-1 brane induced gravity models have been discussed recently in [56].

Note, that our construction is completely covariant from the bulk point of view, since we can define the regulator by a variational principle, from a well defined 4-brane action. Therefore we can explicitly check the properties of brane localized gravity, and calculate the crossover scale inferred in [29] exactly.

\subsection{Stress Energy Sources on Thick Branes}

Having constructed the thick brane vacua, we can turn to the description of matter sources that reside on branes. For simplicity we take the matter to be localized on the 4-brane, and described by a matter sector action

$$
S_{\text {matter }}=-\int d^{5} x \sqrt{g_{5}} \mathcal{L}_{\text {matter }}
$$


where the matter couples to the induced metric on the 4-brane $g_{5 a b}$. Since one of the dimensions along the brane is a circle of radius $r_{0}$, and we are interested in the behavior of the theory at length scales $r \gg r_{0}$, we dimensionally reduce the brane-localized sector on this direction, representing all brane fields by the standard Fourier expansion

$$
\Phi_{\mathcal{N}}\left(x^{\mu}, r_{0} \phi\right)=\sum_{n=-\infty}^{\infty} \Phi_{\mathcal{N}, n}\left(x^{\mu}\right) e^{i n \phi},
$$

where $\mathcal{N}$ represents the quantum numbers of any particular representation on the brane. The Fourier coefficients then give rise to the $4 D \mathrm{KK}$ towers of fields, with masses $M^{2}=m^{2}+n^{2} / r_{0}^{2}$, where $m$ are the explicit mass terms from (29), and the mass gap is $1 / r_{0}$. This expansion also applies to the brane induced metric and its curvature. Thus, at distances $r \gg r_{0}$ we can always model the angular dependence of any configuration as a small, Yukawa-suppressed correction from a KK momentum mode stretching along the compact circle.

When we consider the classical limit of the theory, to define the gravitational sources that describe lumps of matter living on the brane, we can model them as loops of string along the cylinder. At distances $r \gg r_{0}$ these will look just like point particles on a thin 3-brane. To determine the stress energy tensors of such objects, that source their gravity, we replace (29) by a Nambu-Goto action for a string,

$$
S_{\text {matter }}=-\mu \int d^{2} \sigma \sqrt{\gamma}
$$

where $\mu$ is the mass per unit length of the string, $\sigma^{\alpha}$ are its two worldsheet coordinates, which we will gauge fix to be $t$ and $r_{0} \phi$, and $\gamma_{\alpha \beta}$ is the induced worldsheet metric. We then vary this action with respect to the metric of the target space on which the string moves, in this case the metric of the background thick brane. We then use the standard definition of the stress energy tensor $\tau^{a b}$, given by the functional derivative equation $\delta S_{\text {matter }}=\frac{1}{2} \int d^{5} x \sqrt{g_{5}} \delta g_{a b} \tau^{a b}$, to read off the stress energy tensor of the loop. Following the standard tricks for pointlike sources, we first rewrite (31) as

$$
S_{m}=-\mu \int d^{2} \sigma d^{5} x \sqrt{\gamma} \delta^{(5)}\left(x^{M}-x^{M}(\sigma)\right)
$$

where the $\delta$-function puts the string on its shell (i.e. enforces its equation of motion). Using $\gamma_{\alpha \beta}=g_{a b} \frac{\partial x^{a}}{\partial \sigma^{\alpha}} \frac{\partial x^{b}}{\partial \sigma^{\beta}}$ and $\delta \sqrt{\gamma}=\frac{1}{2} \sqrt{\gamma} \gamma^{\alpha \beta} \delta \gamma_{\alpha \beta}$, we find

$$
\tau^{a b}=-\mu \int d^{2} \sigma \frac{1}{\sqrt{g_{5}}} \delta^{(5)}\left(x^{c}-x^{c}(\sigma)\right) \gamma^{\alpha \beta} \frac{\partial x^{a}}{\partial \sigma^{\alpha}} \frac{\partial x^{b}}{\partial \sigma^{\beta}} .
$$

Similar variation with respect to the target space coordinates of the string leads to the string equation of motion, which is $\nabla_{\alpha}\left(g_{a b} \nabla^{\alpha} x^{b}\right)=0$, and is in fact completely equivalent to the string stress energy conservation, $\nabla_{a} \tau^{a b}=0$.

With the gauge choice $\sigma^{0}=x^{0}$ and $\sigma^{1}=r_{0} \phi$, and using the vacuum solution (25) as the target space, we get $\gamma_{\alpha \beta}=\eta_{\alpha \beta}$, and hence the loop of string stress energy tensor becomes

$$
\tau^{a b}=-\mu \int d^{2} \sigma \delta^{(5)}\left(x^{c}-x^{c}(\sigma)\right) \eta^{\alpha \beta} \frac{\partial x^{a}}{\partial \sigma^{\alpha}} \frac{\partial x^{b}}{\partial \sigma^{\beta}} .
$$


While this expression is only valid on the locally flat target (25), as we will only be interested in the linearized gravitational fields (which however are exact for relativistic particles!) this is all we shall need. If we consider static sources, the string representing only the lightest modes must be translationally invariant in the compact direction, because by the discussion above any inhomogeneities along the string can be viewed as heavy KK states at distances $r \gg r_{0}$. For such axially symmetric sources, only two components of (34) survive: $\tau^{00}=\mu \delta^{(3)}(\vec{x})$, and $\tau^{\phi \phi}=-\frac{\mu}{r_{0}} \delta^{(3)}(\vec{x})$, which we can write more compactly as

$$
\tau_{b}^{a}=-\mu \delta^{(3)}(\vec{x}) \operatorname{diag}(1,0,0,0,1) .
$$

The parameter $\mu$ is the total rest mass per unit length of the configuration. To get the stress energy tensor of a relativistic string, that moves along the cylindrical brane at the speed of light, and has vanishing rest mass, we can simply boost (35) along the direction of motion $x_{\|}$, go to the light cone coordinates $x_{\|}=v+u, t=v-u$ and take the limit of infinite boost parameter while sending $\mu \rightarrow 0$ [65]. Since the momentum of the string $p$ is the length of the string $2 \pi r_{0}$ multiplied by the finite limit of $\mu \cosh \beta$ as $\beta$ diverges, for sources composed of the lightest modes as in (35) this yields, thanks to the properties of the vacuum (25),

$$
\tau^{\mu}{ }_{\nu}=\frac{2 p}{2 \pi r_{0} \sqrt{g_{4}}} g_{4 u v} \delta(u) \delta^{(2)}\left(\vec{x}_{\perp}\right) \delta^{\mu}{ }_{v} \delta^{u}{ }_{\nu}, \quad \quad \tau_{\phi}^{\phi}=0,
$$

which is identical in form to the relativistic stress energy on thin branes, used in the previous section.

To find the gravitational fields of these sources, we need to solve the brane induced gravity field equations (20), in Gaussian-normal gauge with the brane at $y=\rho-r_{0}=0$ and with $T_{b}^{a}$, which is given by the sum of the vacuum contribution in Eq. (17) and $\tau^{a}{ }_{b}$ as given in (35) or (36), on the right-hand side. It will turn out to be convenient to rewrite these equations as the conditions on the Ricci tensor, which after tracing over the indices and rearranging the scalar contributions, become

$$
M_{6}^{4} R_{6}{ }_{B}^{A}=\left[\left(T^{a}{ }_{b}-M_{5}^{3}\left(R_{5}{ }^{a}{ }_{b}-\frac{1}{2} \delta^{a}{ }_{b} R_{5}\right)\right) \delta^{A}{ }_{a} \delta^{b}{ }_{B}-\frac{1}{4} \delta^{A}{ }_{B}\left(T^{a}{ }_{a}+\frac{3}{2} M_{5}^{3} R_{5}\right)\right] \delta\left(\rho-r_{0}\right) .
$$

We first consider the relativistic sources obeying (36) and find the generalization of the shock wave solution (5), (14) on thick branes.

\section{Shocking Crossover Physics}

\subsection{Setting Up the Shock Wave}

As before, we determine the shock wave solution by introducing a discontinuity in the metric of Eq. (25) according to the prescription of [65, 16], and demanding that the wave profile solves Eq. (37) with (17) and (36) as sources. We are working with sub-critical branes, $b<1$, which admit static flat vacua. We can still, however, consider the limit $b \rightarrow 1$. Using the null coordinates $u, v$ we require that the source moves along $u=0$, and that the wave 
profile explores all the transverse dimensions available to gravity. By axial symmetry of the source, however, we only need to look at the profiles $f\left(\vec{x}_{\perp}, \rho\right) \delta(u)$ that depend on the transverse coordinates along the brane $\vec{x}_{\perp}$ and the transverse distance $\rho$ orthogonal to the brane. Thus we seek the shock wave metric in the form

$$
d s_{6}{ }^{2}=4 d u d v-4 \delta(u) f d u^{2}+d \vec{x}_{\perp}^{2}+d \rho^{2}+\left[\left(1-b \Theta\left(\rho-r_{0}\right)\right) \rho+b r_{0} \Theta\left(\rho-r_{0}\right)\right]^{2} d \phi^{2}
$$

Now, we substitute the sum of Eqs. (17) and (36) along with the condition (18) in Eq. (37) and trace over the indices. We note that the stress energy of a relativistic particle (36) is traceless, reflecting that the source is scale-free. We can then easily check that by the form of the shock wave metric (38) the trace of the $6 D$ Ricci tensor picks up contributions only from the $\rho$ - $\phi$ part of the metric, $R_{6}=R_{2}$, leading to an equation identical to Eq. (21). Thus as before this equation is trivially satisfied by Eq. (38), with the deficit angle parameter defined in (23), and so are all the tensor equations on the truncated cone $\rho-\phi$.

Among the remaining tensor equations of (37), the only one which contains nontrivial information, as before, is $\propto R_{6}{ }^{v}{ }_{u}$. Indeed, Eqns. (37) on the background (38) reduce to

$$
M_{6}^{4} R_{6}{ }^{\mu}{ }_{\nu}+M_{5}^{3} R_{5}{ }^{\mu}{ }_{\nu} \delta\left(\rho-r_{0}\right)=\tau^{\mu}{ }_{\nu} \delta\left(\rho-r_{0}\right)
$$

and the only components of the Ricci tensors which depends on the shock wave profile are

$$
\begin{aligned}
& R_{6}{ }^{v}{ }_{u}=\delta(u) \nabla_{4}{ }^{2} f, \\
& R_{5}{ }^{v}{ }^{u}=\delta(u) \nabla_{2}{ }^{2} f,
\end{aligned}
$$

where we are explicitly using axial symmetry in the latter term when we write the Laplacian in only the two transverse spatial coordinates $\vec{x}_{\perp}$. Substituting this and (36) in Eq. (39), after a straightforward algebra we finally get

$$
\nabla_{4}^{2} f+\frac{M_{5}^{3}}{M_{6}^{4}} \nabla_{2}^{2} f \delta\left(\rho-r_{0}\right)=\frac{2 p}{2 \pi r_{0} M_{6}^{4}} \delta^{2}\left(\vec{x}_{\perp}\right) \delta\left(\rho-r_{0}\right) .
$$

This equation is almost identical to Eq. (8) governing the shock wave profile on a thin 3 -brane. The main difference is that the $\delta$-function is shifted from the origin and smeared over a circle $\rho=r_{0}$, and that the Laplacian $\nabla_{4}^{2}$ is defined on the truncated cone (25), such

that for axially symmetric shocks, $\nabla_{4}^{2} f=\nabla_{\vec{x}_{\perp}}^{2} f+f^{\prime \prime}+\frac{1-b \Theta\left(\rho-r_{0}\right)}{\left(1-b \Theta\left(\rho-r_{0}\right)\right) \rho+b r_{0} \Theta\left(\rho-r_{0}\right)} f^{\prime}$, where the prime denotes the $\rho$-derivative. The latter term contains a jump across the 4 -brane.

\subsection{Solving for the Shock Wave Profile}

To solve (41), we again Fourier transform in the brane transverse space, using Eq. (9). This yields

$$
\left(\nabla_{\vec{y}}^{2}-k^{2}\right) \varphi_{k}=\left(\frac{2 p}{2 \pi r_{0} M_{6}^{4}}+\frac{M_{5}^{3}}{M_{6}^{4}} k^{2} \varphi_{k}\right) \delta\left(\rho-r_{0}\right) .
$$

In contrast to Eq. (8) here we have a single $\delta$-function. Of course, this is because this time the brane is codimension-1. Hence we can use the standard methods for finding bulk field 
wave functions in the codimension-1 brane setups: we solve Eq. (42) inside (-) and outside $(+)$ the brane, and then use the boundary conditions, which are the continuity of $\varphi_{k}$, the jump of $\varphi_{k}^{\prime}$ as prescribed by Gaussian pillbox integration of (42) around the brane, and the regularity of the solution in the center of (25) and at infinity:

$$
\begin{aligned}
\varphi_{k}^{-}(0) & =0 \\
\varphi_{k}^{+}(\infty) & =0 \\
\varphi_{k}^{+}\left(r_{0}\right) & =\varphi_{k}^{-}\left(r_{0}\right), \\
\varphi_{k}^{+\prime}\left(r_{0}\right)-\varphi_{k}^{-\prime}\left(r_{0}\right) & =\left(\frac{2 p}{2 \pi r_{0} M_{6}^{4}}+\frac{M_{5}^{3}}{M_{6}^{4}} k^{2} \varphi_{k}\left(r_{0}\right)\right) .
\end{aligned}
$$

Away from the brane the differential equation (42) gives

$$
\varphi_{k}^{\prime \prime}+\frac{1}{\rho+\frac{b r_{0} \Theta\left(\rho-r_{0}\right)}{1-b \Theta\left(\rho-r_{0}\right)}} \varphi_{k}{ }^{\prime}-k^{2} \varphi_{k}=0
$$

with Bessel functions $I_{0}\left(k\left(\rho+\frac{b r_{0} \Theta\left(\rho-r_{0}\right)}{1-b \Theta\left(\rho-r_{0}\right)}\right)\right), K_{0}\left(k\left(\rho+\frac{b r_{0} \Theta\left(\rho-r_{0}\right)}{1-b \Theta\left(\rho-r_{0}\right)}\right)\right)$ as solutions. The first two of the boundary conditions in Eq. (43) then pick $\varphi_{k}^{-} \sim I_{0}, \varphi_{k}^{+} \sim K_{0}$, and the third condition sets $\varphi_{k}^{-}=A I_{0}(k \rho) K_{0}\left(k \frac{r_{0}}{1-b}\right)$ and $\varphi_{k}^{+}=A I_{0}\left(k r_{0}\right) K_{0}\left(k\left(\rho+\frac{b r_{0}}{1-b}\right)\right)$. Finally the last of $(43)$ fixes the coefficient $A$, yielding

$$
A=-\frac{2 p}{2 \pi r_{0}\left[M_{6}^{4} k\left(I_{0}\left(k r_{0}\right) K_{1}\left(k \frac{r_{0}}{1-b}\right)+I_{1}\left(k r_{0}\right) K_{0}\left(k \frac{r_{0}}{1-b}\right)\right)+M_{5}^{3} k^{2} I_{0}\left(k r_{0}\right) K_{0}\left(k \frac{r_{0}}{1-b}\right)\right]},
$$

where we have used the recursion formulas for modified Bessel functions $\partial_{z} I_{0}(z)=I_{1}(z)$ and $\partial_{z} K_{0}(z)=-K_{1}(z)$. Substituting this and the form of the solutions $\varphi_{k}$ back in the Fourier transform equations (9), and again using the axial symmetry in the $\vec{x}_{\perp}$ plane to integrate over the transverse spatial angle, yielding $f=\frac{1}{2 \pi} \int_{0}^{\infty} d k k \varphi_{k} J_{0}\left(k\left|\vec{x}_{\perp}\right|\right)$, we finally obtain the expression for the shock wave profile on the truncated cone:

$$
f\left(\vec{x}_{\perp}, \rho\right)=-\frac{p}{2 \pi^{2} r_{0}} \int_{0}^{\infty} d k \frac{I_{0}\left(k \rho_{<}\right) K_{0}\left(k \rho_{>}\right) J_{0}\left(k\left|\vec{x}_{\perp}\right|\right)}{M_{6}^{4}\left(I_{0}\left(k r_{0}\right) K_{1}\left(k \frac{r_{0}}{1-b}\right)+I_{1}\left(k r_{0}\right) K_{0}\left(k \frac{r_{0}}{1-b}\right)\right)+M_{5}^{3} k I_{0}\left(k r_{0}\right) K_{0}\left(k \frac{r_{0}}{1-b}\right)},
$$

where

$$
I_{0}\left(k \rho_{<}\right) K_{0}\left(k \rho_{>}\right)=\left\{\begin{array}{ll}
I_{0}(k \rho) K_{0}\left(k \frac{r_{0}}{1-b}\right), & \rho \leq r_{0} \\
I_{0}\left(k r_{0}\right) K_{0}\left(k\left(\rho+\frac{b r_{0}}{1-b}\right)\right), & \rho \geq r_{0}
\end{array} .\right.
$$

This solution is clearly not divergent away from the brane, unlike (8), as long as $r_{0}>0$. Indeed, along the brane, $\rho=r_{0}$, it reduces to

$$
f\left(\vec{x}_{\perp}, r_{0}\right)=-\frac{p}{2 \pi^{2} r_{0}} \int_{0}^{\infty} d k \frac{I_{0}\left(k r_{0}\right) K_{0}\left(k \frac{r_{0}}{1-b}\right) J_{0}\left(k\left|\vec{x}_{\perp}\right|\right)}{M_{6}^{4}\left(I_{0}\left(k r_{0}\right) K_{1}\left(k \frac{r_{0}}{1-b}\right)+I_{1}\left(k r_{0}\right) K_{0}\left(k \frac{r_{0}}{1-b}\right)\right)+M_{5}^{3} k I_{0}\left(k r_{0}\right) K_{0}\left(k \frac{r_{0}}{1-b}\right)}
$$

where all the individual contributions remain finite. 


\subsection{Crossover Physics}

Now we turn to the physics of the solution (46)-(48). It is subtle, controlled by the deficit angle of the background (25). Let us look at the field along the brane, (48). The shock wave profile in (48) is dominated by the modes with the momenta $k \sim 1 /\left|\vec{x}_{\perp}\right|$, due to the oscillatory nature of $J_{0}$. The contributions of the modes with momenta $k$ far from $1 /\left|\vec{x}_{\perp}\right|$ will interfere destructively. At transverse distances much larger than the size of the compact dimension, $\left|\vec{x}_{\perp}\right| \gg r_{0}$, we need to focus on the momenta for which $k r_{0} \ll 1$. Thus we can always replace the terms $\propto I_{n}\left(k r_{0}\right)$ by their small argument expansion. For sub-critical branes, $1-b \sim \mathcal{O}(1)$, and we can likewise replace any $K_{n}\left(k \frac{r_{0}}{1-b}\right)$ by their small argument expansion too. However, in the near-critical limit the deficit angle approaches $2 \pi$ and so $|1-b| \ll 1$. Hence the argument of $K_{0}\left(k \frac{r_{0}}{1-b}\right)$ may be very large even when $k r_{0} \ll 1$. Thus we must consider the near-critical branes very carefully.

We start with sub-critical branes where $k r_{0} \ll 1-b$, and we can approximate all Bessel functions by their small argument form. Using $I_{\nu}(z) \rightarrow \frac{1}{\Gamma(\nu+1)}\left(\frac{z}{2}\right)^{\nu}, K_{\nu}(z) \rightarrow \frac{\Gamma(\nu)}{2}\left(\frac{2}{z}\right)^{\nu}$ for $\nu>0$ and $K_{0}(z) \rightarrow \ln (2 / z)$, the shock along the brane then becomes

$$
f\left(\vec{x}_{\perp}, r_{0}\right) \simeq-\frac{p}{2 \pi^{2} M_{6}^{4}} \int_{0}^{\infty} d k k \frac{\ln \left[\frac{2(1-b)}{k r_{0}}\right] J_{0}\left(k\left|\vec{x}_{\perp}\right|\right)}{(1-b)+\frac{M_{5}^{3} r_{0}}{M_{6}^{4}} k^{2} \ln \left[\frac{2(1-b)}{k r_{0}}\right]} .
$$

If $k \sim 1 /\left|\vec{x}_{\perp}\right|$ is large enough for the second term in the denominator to dominate, which in this approximation - happens when

$$
k^{2}>k_{c}^{2}(k)=\frac{(1-b) M_{6}^{4}}{M_{5}^{3} r_{0} \ln \left[\frac{2(1-b)}{k r_{0}}\right]},
$$

the logs in the integrand cancel, and the shock wave profile is

$$
f\left(\vec{x}_{\perp}, r_{0}\right) \simeq-\frac{p}{2 \pi^{2} M_{5}^{3} r_{0}} \int_{k_{c}}^{\infty} d k \frac{J_{0}\left(k\left|\vec{x}_{\perp}\right|\right)}{k}=\frac{p}{\pi M_{4}^{2}} \ln \left(k_{c}\left|\vec{x}_{\perp}\right|\right)+\ldots
$$

where we have used Gauss law to normalize the lightest KK modes in the expansion of the brane induced curvature on the circle of radius $r_{0}$ to $M_{4}^{2}=2 \pi M_{5}^{3} r_{0}$. This is - to the leading order - precisely the $4 D$ Aichelburg-Sexl solution, with the log profile normalized to the inverse of the critical momentum $k_{c}$ given in (50), as opposed to some arbitrary short distance cutoff $\ell$.

For sub-critical branes when $1-b \sim \mathcal{O}(1)$, this is the end of the $4 D$ story: all distances longer than $r_{0}$ fall in the $k r_{0} \ll 1$ regime, and the gravitational field looks $4 D$, like (51), only when $r<r_{c}=1 / k_{c}$. In this, generic, case, the crossover scale is qualitatively very different from the see-saw scale of [29]. Indeed, using Gauss law $M_{5}^{3}=\frac{M_{4}^{2}}{2 \pi r_{0}}$, we can rewrite $1 / k_{c}^{2}$ from (50) as

$$
r_{c}^{2}(k)=\frac{M_{4}^{2}}{2 \pi(1-b) M_{6}^{4}} \ln \left[\frac{2(1-b)}{k r_{0}}\right],
$$

or, ignoring the $\log , r_{c} \sim \frac{M_{4}}{M_{6 \text { eff }}^{2}}$, where $M_{6 \text { eff }}^{4}=(1-b) M_{6}^{4}$. Thus for sub-critical branes the crossover scale is set by the 'naive' ratio of brane and effective bulk Planck scales, and 
depends on the UV cutoff $r_{0}$ of the brane core only through the logarithmic correction reminiscent of 'running' in real space, which was discussed for locally localized gravity in [63]. This shows that the crossover weakly depends on the momentum, and is very different from the see-saw mechanism of [29]. We also see that the bulk gravitational coupling $1 / M_{6}^{4}$ is amplified by the 'lightning rod' factor $(1-b)^{-1}$ of [50]. This 'lightning rod' amplification is intuitively clear, because gravitational field lines spread more slowly on a cone than on a plane, at a fixed distance from a source, and hence gravity must look stronger on a cone [50]. It plays an even more important role in the near-critical limit, as we will see below.

At distances larger than $r_{c}$, gravity eventually changes to $6 D$. For sub-critical branes, this transition actually looks like a rapid 'running' of the coupling of individual graviton modes in the resonance that impersonates General Relativity. We can glimpse this, for example, from Eq. (49), which shows that the effective coupling controlling the transfer of the momentum $k$ is

$$
\frac{1}{M_{6 \mathrm{eff}}^{4}}=\frac{1}{(1-b) M_{6}^{4}} \ln \left[\frac{2(1-b)}{k r_{0}}\right] .
$$

In contrast to the $5 D$ case [16], the running here is logarithmic, because there are two extra dimensions as probed by the localized masses on the wrapped brane. To recover the full $6 D$ form of the wave, we can take (47) or (48), and taking the limit of very large distance, ignore the second term in the denominators of these integrals. In this case,

$$
f \simeq-\frac{p}{2 \pi^{2}(1-b) M_{6}^{4}} \int_{0}^{\infty} d k k I_{0}\left(k r_{0}\right) K_{0}\left(k\left(\rho+\frac{r_{0}}{1-b}\right)\right) J_{0}\left(k\left|\vec{x}_{\perp}\right|\right)+\ldots
$$

where the integral can be done in closed form, leading to [50]

$$
f \simeq-\frac{p}{2 \pi^{2}(1-b) M_{6}^{4}} \frac{1}{\left|\vec{x}_{\perp}\right|^{2}+\rho^{2}}+\ldots
$$

This is the $6 D$ gravitational shock wave, constructed in [58], with the 'lightning rod' term accounting for the brane tension as found in [50].

Let us now consider the near-critical limit, $b \rightarrow 1$, which requires particular care. In this limit, Eq. (52) suggests that the crossover scale diverges, indicating that gravity remains $4 D$ out to extremely large distances, regardless of the brane thickness. However our discussion of the near-critical limit in Sec. (3), in particular interpretation of Eq. (27), has already taught us otherwise. As we have seen there, in the near-critical limit the bulk around the brane compactifies to a long cylinder, which opens up into a cone only very far from the brane. Bulk gravity in the throat is in the $5 D$ regime, which separates the $4 D$ and $6 D$ ones. Thus the crossover scale beyond which gravity is not $4 D$ is really the demarkation between the $4 D$ regime, and $5 D$ gravity which lives inside the throat. Looking at the shock wave solution (48), we see that while the small argument expansion for $I_{n}(z)$ always suffices for large distances from the source, in the near-critical limit we must treat the functions $K_{n}(z)$ carefully for the momenta in the regime $1-b \ll k r_{0} \ll 1$. Indeed, this corresponds to length scales $\ell \lesssim \frac{r_{0}}{1-b}$ which are shorter than the length of the conical throat. So setting $I_{0}\left(k r_{0}\right) \rightarrow 1$ and $I_{1}\left(k r_{0}\right) \stackrel{\rightarrow}{\rightarrow} 0$ in (48), we notice that where the terms $\propto M_{5}^{3}$ dominate, gravity will remain $4 D$ because the factors of $K_{0}\left(k \frac{r_{0}}{1-b}\right)$ exactly cancel in the integrand. This happens for the 
momenta $k$ which satisfy $k>\frac{M_{6}^{4}}{M_{5}^{3}} \frac{K_{1}\left(k \frac{r_{0}}{1-b}\right)}{K_{0}\left(\frac{\nu_{0}}{1-b}\right)}$. In the near-critical limit this can occur when the argument of Bessel functions is much larger than unity, so that the ratio $K_{1} / K_{0}$ will be of the order of unity! Indeed, as we decrease $k r_{0}$, going out to larger distances, $K_{1}$ will remain close to $K_{0}$ : since $K_{1}(z)=-\partial_{z} K_{0}(z)$ and now, since $b \rightarrow 1$, we have large values of the argument for which $K_{0}(z) \rightarrow \sqrt{\frac{\pi}{2 z}} e^{-z}$, so that $K_{1} \rightarrow K_{0}$. The transition can occur only once, because $K_{n}$ are monotonic, and for our approximation to be valid it must happen when $\frac{k r_{0}}{1-b} \geq \mathcal{O}(1)$ - otherwise the situation will be essentially the same as in a generic sub-critical case. In that case the crossover scale saturates to $k_{c}=\frac{M_{6}^{4}}{M_{5}^{3}}$, or therefore

$$
r_{c}=\frac{M_{5}^{3}}{M_{6}^{4}}
$$

Clearly, this is valid as long as $r_{c}<\frac{r_{0}}{1-b}$. The scale $r_{c}$ is exactly the see-saw scale of [29]: again using Gauss's law for the $4 D$ Planck mass, $M_{4}^{2}=2 \pi M_{5}^{3} r_{0}$, we find

$$
r_{c}=\frac{M_{4}^{2}}{2 \pi M_{6}^{4} r_{0}} .
$$

This demystifies the appearance of the see-saw effect, showing that at least with our regularization, it does happen naturally, but only for near-critical branes whose deficit angle is very close to $2 \pi$, so that they still are flat and static.

As an explicit check that inside the conical throat gravity looks $5 D$, we can take the limit of our solution for a fixed distance $\left|\vec{x}_{\perp}\right|$ and formally take the limit $b \rightarrow 1$ while holding $r_{0}$ fixed. Then if we take the solution in Eq. (48), in the limit $b \rightarrow 1$ we can replace $K_{n}$ 's by their large argument expansion for any finite momentum. After cancelling the like terms, and taking the thin brane limit, we find, using footnote (2),

$$
f\left(\vec{x}_{\perp}, \rho\right)=-\frac{p}{2 \pi^{2}} \int_{0}^{\infty} d k \frac{J_{0}\left(k\left|\vec{x}_{\perp}\right|\right)}{M_{6}^{4} r_{0}+M_{5}^{3} r_{0} k}=-2 p \int_{-\infty}^{\infty} \frac{d^{2} \vec{k}}{(2 \pi)^{2}} \frac{e^{i \vec{k} \cdot \vec{x}_{\perp}}}{2 \pi M_{6}^{4} r_{0} k+2 \pi M_{5}^{3} r_{0} k^{2}} .
$$

This is precisely the shock wave profile on a flat thin 3-brane in $5 D$, on the normal branch, with brane and bulk Planck masses $M_{4 \text { eff }}^{2}=2 \pi M_{5}^{3} r_{0}$ and $M_{5 \text { eff }}^{3}=2 \pi M_{6}^{4} r_{0}$, respectively. It can be obtained as a limit of the normal branch solutions of [16] on a tensionless brane.

So to sum up, we find that for distances $r_{0}<r<r_{c}$ the shock wave reduces to the Aichelburg-Sexl solution [57], and becomes its higher-dimensional generalization when $r>r_{c}$. The crossover scale for sub-critical and near-critical branes, beyond which gravity is not $4 D$ anymore, is given by

$$
r_{c}^{2}= \begin{cases}\frac{M_{4}^{2}}{2 \pi(1-b) M_{6}^{4}} \ln \left[\frac{2(1-b)}{k r_{0}}\right], & b \lesssim 1 ; \\ \frac{M_{4}^{4}}{4 \pi^{2} M_{6}^{8} r_{0}^{2}}, & b \rightarrow 1,\end{cases}
$$

only in the latter case agreeing with the see-saw scale discussed in [29]. The origin of the see-saw mechanism is now clear: in the near-critical limit, the brane resides in a very deep throat generated by its own tension. Thus its localized $4 D$ gravity first changes to $5 D$ gravity residing inside the throat, and only later to full $6 D$ gravity [59], at distances $\rho \gg r_{0} /(1-b)$ (see discussion of Eq. (27)). This shows how the see-saw mechanism emerges. 


\section{$5 \quad$ Linearized Gravity}

\subsection{Perturbations and Their Gauge Symmetries}

We can solve exactly the field equations (1) for localized matter sources only in the relativistic limit. For nonrelativistic sources, we can try to solve them perturbatively, assuming that the solutions converge to the background vacuum far from the sources. So starting with a background $d s_{6}^{2}=g_{A B} d x^{A} d x^{B}$ metric, we define the metric perturbations $h_{A B}$ as is usual, by the substitution $g_{A B} \rightarrow g_{A B}+h_{A B}$. In addition, we must also perturb the brane location $r_{0} \rightarrow r_{0}+\xi$ and any nontrivial field configurations on the brane, in our case the axion $\Sigma \rightarrow$ $\Sigma+\sigma$. Before substituting these expansions in the field equations (1), we must account for the gauge symmetries of the perturbations, to identify physically meaningful variables which cannot be undone with diffeomorphisms. So suppose that we transform $x^{A} \rightarrow \bar{x}^{A}=x^{A}+\chi^{A}$. A straightforward calculation then shows that the perturbations transform according to

$h_{A B} \rightarrow \bar{h}_{A B}=h_{A B}-\nabla_{A} \chi_{B}-\nabla_{B} \chi_{A}, \quad \xi \rightarrow \bar{\xi}=\xi+\left.\chi^{\rho}\right|_{\rho=r_{0}}, \quad \sigma \rightarrow \bar{\sigma}=\sigma-\left.\chi^{A}\right|_{\rho=r_{0}} \nabla_{A} \Sigma$,

where the $\nabla_{A}$ and raising and lowering of indices are defined with respect to the background metric $g_{A B}$. On the static thick brane background (25), we can immediately compute the transformations (60), splitting them up with respect to different representations of the $4 D$ Lorentz symmetry. They are

$$
\begin{aligned}
\bar{h}_{\mu \nu} & =h_{\mu \nu}-\partial_{\mu} \chi_{\nu}-\partial_{\nu} \chi_{\mu}, \\
\bar{h}_{\phi \phi} & =h_{\phi \phi}-2 \partial_{\phi} \chi_{\phi}-\alpha^{\prime} \chi^{\rho}, \\
\bar{h}_{\mu \phi} & =h_{\mu \phi}-\partial_{\mu} \chi_{\phi}-\partial_{\phi} \chi_{\mu}, \\
\bar{h}_{\mu \rho} & =h_{\mu \rho}-\partial_{\mu} \chi^{\rho}-\chi_{\mu}{ }^{\prime}, \\
\bar{h}_{\rho \phi} & =h_{\rho \phi}-\partial_{\phi} \chi^{\rho}-\chi_{\phi}{ }^{\prime}+\chi_{\phi} \frac{\alpha^{\prime}}{\alpha}, \\
\bar{h}_{\rho \rho} & =h_{\rho \rho}-2 \chi^{\rho \prime} \\
\bar{\xi} & =\xi+\chi^{\rho}\left(r_{0}\right) \\
\bar{\sigma} & =\sigma-q \chi^{\phi}\left(r_{0}\right)
\end{aligned}
$$

where

$$
\alpha=g_{\phi \phi}=\left(\left(1-b \Theta\left(\rho-r_{0}\right)\right) \rho+b r_{0} \Theta\left(\rho-r_{0}\right)\right)^{2},
$$

is the area of the disk at fixed $\rho$ in Eq. (25), and $\chi^{\rho}\left(r_{0}\right)=\left.\chi^{\rho}\right|_{\rho=r_{0}}$. We need to carefully raise and lower the angular index $\phi$, since although the brane is intrinsically flat, the full geometry isn't. Note, that while $\alpha$ is continuous, $\frac{\alpha^{\prime}}{\sqrt{\alpha}}=2\left(1-b \Theta\left(\rho-r_{0}\right)\right)$, so that on the brane, the discontinuity, defined for any function as the jump of its value across the brane, $\Delta f=\left.f\right|_{+}-\left.f\right|_{-}=f\left(r_{0}{ }^{+}\right)-f\left(r_{0}^{-}\right)$, is $\Delta \frac{\alpha^{\prime}}{\alpha}=-4 \frac{\lambda_{5}}{M_{6}^{4}}$, where we have used Eq. (23).

Using these transformations we can always gauge fix the metric perturbed by axially symmetric sources to brane-fixed Gaussian-normal gauge, in the linear order. The key is to be careful about what axial symmetry means: the vector field $\partial_{\phi}$ must be a Killing vector of the metric, unperturbed as well as perturbed. Hence any tensor, including the metric, 
must be Lie-derived by $\partial_{\phi}$ to zero. Now, using (61), we can readily pick $\chi^{\rho}$ and $\chi_{\phi}$ to set $\bar{h}_{\rho \rho}$ and $\bar{h}_{\rho \phi}$ to zero, respectively, treating the corresponding transformation rules in (61) as differential equations defining $\chi^{\rho}$ and $\chi_{\phi}$. We can always choose the boundary condition $\chi^{\rho}\left(r_{0}\right)=0$, opting to keep the perturbation of the brane location $\xi$, or brane bending, to remain unchanged, for the moment. Next, we can pick a $\chi_{\mu}=\chi_{\mu}(\rho, \phi)$ to set $\bar{h}_{\mu \rho}$ and $\bar{h}_{\mu \phi}$ to zero too. This may seem to induce angular dependence of $\bar{h}_{\mu \nu}$ by the first of Eqs. (61). However, it's easy to check that in the coordinates where $\bar{h}_{\rho \rho}=\bar{h}_{\rho \phi}=\bar{h}_{\mu \phi}=\bar{h}_{\mu \rho}=0$ the Killing conditions enforcing axial symmetry imply that $\partial_{\phi} \bar{h}_{\mu \nu}=\partial_{\phi} \bar{h}_{\phi \phi}=0$. Thus the metric perturbations $\bar{h}_{A B}$ are $\phi$-independent. In fact, this proof extends beyond linear perturbation theory, as the reader can check by consulting [64]. Notice that we have now completely used up $\chi^{\phi}\left(r_{0}\right)$ - we can't change $\sigma$ without also changing $h_{\rho \phi}$, as is clear from (61). This shows that the lightest in the tower of the KK vectors $h_{\rho a}$ eats the brane-localized axion $\sigma$ to become massive. Alternatively, when we pick the gauge $h_{\rho a}=0$, as we did here, we are separating out the scalar $\sigma$ as the Stückelberg field of $h_{\rho a}$.

Dropping the overbars, note that in any Gaussian-normal gauge $h_{\rho \rho}=h_{\rho \phi}=h_{\mu \phi}=$ $h_{\mu \rho}=0$, we still have the residual gauge freedom generated by $\chi_{\mu}$, which are independent of $\rho$ and $\phi$, and by $\chi^{\rho}\left(r_{0}\right)$. These transformations do not change our gauge. They do change $h_{\mu \nu}, h_{\phi \phi}, \xi$. Transformations generated by $\chi^{\mu}$ are the standard $4 D$ diffeomorphisms, and we will find their uses later on. Transformations generated by $\chi^{\rho}\left(r_{0}\right)$ allow us to remove the brane bending, nailing the brane straight to its background value, $\rho=r_{0}$. In this gauge we can quickly find the field equations for the perturbations, by directly substituting the gauge-fixed perturbed metric into the field equations (1) or (37).

Going to this gauge requires a little care, however. As we have noted above, $\frac{\alpha^{\prime}}{\alpha}$ is discontinuous on the brane in the background (25). Hence when $\chi^{\rho}\left(r_{0}\right) \neq 0$, the gauge transformation (61) changes the metric perturbation $h_{\phi \phi}$ discontinuously. On the other hand, in brane-fixed Gaussian-normal gauge the full field configuration has discontinuities on the straight brane, proportional to its the stress-energy. These must remain encoded in the $\delta$-function sources in (37). Hence the metric perturbations in brane-fixed Gaussian-normal gauge must be continuous across the brane, and their derivatives may have at most a finite jump there. In any other gauge found by using a transformation (61) with $\chi^{\rho}\left(r_{0}\right) \neq 0$, the metric perturbation $h_{\phi \phi}$ will appear discontinuous, so that the brane matching conditions on the perturbations can only be found by carefully implementing the Israel junction conditions (for an illustration, see e.g. [51]). So to get the field equations, we choose to work in branefixed Gaussian-normal gauge, where we get the brane matching conditions by using Gaussian pillbox integration around the brane, and relating the normal derivative discontinuities to $\delta$-sources in the field equations. This discussion applies completely generally to any model where a codimension- 2 brane is resolved by a cylindrical codimension-1 brane.

Nevertheless, it turns out the bulk field equations for perturbations are easier to solve by stepping out of the brane-fixed Gaussian-normal gauge, to a gauge where the $\rho$ - $\phi$ cone appears conformally flat, and explicitly keeping the brane bending $\xi$. The latter is similar to what happens in other codimension-1 cases [67], except that here the brane bending must be turned on even in the $4 D$ vacuum (25). The reason is that the $4 D$ vacuum backgrounds (25) are not codimension-1 vacua: the axion flux breaks the symmetry between the longitudinal brane directions. Brane bending probes this 'vacuum' anisotropy. Using the conformally 
flat $\rho-\phi$ metric, on the other hand, simplifies the bulk field equations. For later use, we list here how to relate such gauges. In a Gaussian-normal gauge the only nonvanishing metric perturbation is $\hat{h}_{\phi \phi}$, now denoted with the caret. Defining $\hat{\Phi}$ by $\hat{h}_{\phi \phi}=\alpha \hat{\Phi}$, the perturbed metric is

$$
d s_{6}{ }^{2}=\left(\eta_{\mu \nu}+h_{\mu \nu}\right) d x^{\mu} d x^{\nu}+d \rho^{2}+(1+\hat{\Phi}) \alpha(\rho) d \phi^{2},
$$

with $\alpha$ defined in (62). Let the brane bending term $\xi$ in this gauge be non-zero. To go to a gauge where it is zero, we apply $(61)$, choosing $\chi^{\rho}\left(r_{0}\right)=-\xi$. That takes us to the gauge $\tilde{\xi}=0$, where the metric has the same form as $(63)$, but with

$$
\tilde{\Phi}=\hat{\Phi}+\frac{\alpha^{\prime}}{\alpha} \xi
$$

instead of $\hat{\Phi}$. This shows how $\tilde{\Phi}$ 'ate' $\xi$ in brane-fixed Gaussian-normal gauge. On the other hand, to relate a general Gaussian-normal gauge to a conformal gauge, we use the relationship

$$
\hat{\Phi}=\Phi-\frac{\alpha^{\prime}}{2 \alpha} \int_{r_{0}}^{\rho} d \rho \Phi
$$

which is a gauge transformation of the form (61) with $\chi^{\rho}=\frac{1}{2} \int_{r_{0}}^{\rho} d \rho \Phi$, and other gauge parameters set to zero. For this gauge transformation, $\chi^{\rho}\left(r_{0}\right)=0$, and therefore it does not change the discontinuities of the perturbations in different gauges. Now, to see which gauge this transformation takes us from, we need to invert this integral equation, and determine $\Phi$. That is straightforward. By definition of $\chi^{\rho}$, we have $\Phi=\frac{1}{2} \chi^{\rho^{\prime}}$, so we can view (65) as a differential equation for $\chi^{\rho}$, with the boundary condition $\chi^{\rho}\left(r_{0}\right)=0$. The equation is $\chi^{\rho \prime}-\frac{\alpha^{\prime}}{2 \alpha} \chi^{\rho}=\hat{\Phi}$, and the solution satisfying the boundary condition is $\chi^{\rho}=\sqrt{\alpha} \int_{r_{0}}^{\rho} d \rho \frac{\hat{\Phi}}{\sqrt{\alpha}}$. Thus

$$
\Phi=\frac{1}{2} \frac{d}{d \rho}\left(\sqrt{\alpha} \int_{r_{0}}^{\rho} d \rho \frac{\hat{\Phi}}{\sqrt{\alpha}}\right) .
$$

Then (65) and (61) imply that in the original gauge $h_{\phi \phi}=\sqrt{\alpha} \Phi$. Furthermore, from $\hat{h}_{\rho \rho}=0$ and Eqs. (65) and $\Phi=\frac{1}{2} \chi^{\rho^{\prime}}$ we see that $h_{\rho \rho}=\sqrt{\alpha} \Phi$ too. So indeed, the gauge transformation (61) defined by (65), (66) relates the general Gaussian-normal perturbed metric (63) to the metric

$$
d s_{6}^{2}=\left(\eta_{\mu \nu}+h_{\mu \nu}\right) d x^{\mu} d x^{\nu}+(1+\Phi)\left(d \rho^{2}+\alpha(\rho) d \phi^{2}\right)
$$

If in addition we want to relate brane-fixed Gaussian-normal gauge perturbation to the $2 D$ conformal gauge perturbation (67), we can combine together the two cases. Then, (64) and (65) yield

$$
\tilde{\Phi}=\Phi-\frac{\alpha^{\prime}}{2 \alpha} \int_{r_{0}}^{\rho} d \rho \Phi+\frac{\alpha^{\prime}}{\alpha} \xi
$$

relating the metric perturbation $\Phi$ in a $2 D$ conformal gauge with brane bending $\xi$ to the metric perturbation $\tilde{\Phi}$ in brane-fixed Gaussian-normal gauge. Since $\tilde{\Phi}$ must be continuous on the brane, we see from (68) that $\Phi$ is not. Instead, it jumps across the brane by $\Delta \Phi=$ $-\Delta\left(\frac{\alpha^{\prime}}{\alpha}\right) \xi=4 \frac{\lambda_{5}}{M_{6}^{4}} \xi$. These formulae will come in handy later on. 


\subsection{Linearized Field Equations and Helicity Decomposition}

We find the field equations for perturbations with axially symmetric sources by substituting the perturbed metric in the brane-fixed Gaussian-normal gauge,

$$
d s_{6}{ }^{2}=\left(\eta_{\mu \nu}+h_{\mu \nu}\right) d x^{\mu} d x^{\nu}+d \rho^{2}+(1+\tilde{\Phi}) \alpha(\rho) d \phi^{2}
$$

with $\tilde{\xi}=0$, into $(37)$, and expanding to the linear order in the perturbation $\tilde{\Phi}$. Since the field equation $\nabla_{a} \nabla^{a} \Sigma=0$ reduces to the source-free $4 D$ Klein-Gordon equation for the axion perturbation $\sigma: \partial_{4}{ }^{2} \sigma=0$, in the linear order the axion is not sourced by axially symmetric matter distributions. We can simply set $\sigma$ to zero from now on. This is self-consistent at distances $|\vec{x}|, \rho \gg r_{0}$. In this limit we can dimensionally reduce the brane theory to a set of KK towers with a mass gap $\propto 1 / r_{0}$. Since a background on the brane which is not axially symmetric must involve higher-level KK states, to encode the angular variation around the brane, at low energies $E \ll 1 / r_{0}$ we can only excite the lightest states, and the backgrounds will automatically be axially symmetric. In fact, since the axion $\sigma$ really is the Stückelberg field of the vector $h_{\rho a}$, it will only be turned on when the vector field is turned on, which means when there is a non-trivial current that can source $h_{\rho a}$.

Evaluating the curvature tensors and the stress-energy terms in (37) we find the field equations for perturbations in this limit. They are the momentum constraint, coming from the $\rho-\mu$ components,

$$
\partial_{\lambda}\left(h_{\mu}^{\lambda \prime}-\delta_{\mu}^{\lambda}\left(h_{4}^{\prime}+\tilde{\Phi}^{\prime}+\frac{\alpha^{\prime}}{2 \alpha} \tilde{\Phi}\right)\right)=0
$$

the trace equation,

$$
h_{4}^{\prime \prime}+\tilde{\Phi}^{\prime \prime}+\frac{\alpha^{\prime}}{\alpha} \tilde{\Phi}^{\prime}=\frac{1}{2 M_{6}^{4}}\left(\tau_{a}^{a}-\frac{3 M_{5}^{3}}{2} \partial_{4}{ }^{2} \tilde{\Phi}+3 \lambda_{5} \tilde{\Phi}+\frac{3 M_{5}^{3}}{2}\left(\partial^{\lambda} \partial_{\sigma} h_{\lambda}^{\sigma}-\partial_{4}{ }^{2} h_{4}\right)\right) \delta\left(\rho-r_{0}\right),
$$

the 'radion field' equation, coming from the $\phi$ - $\phi$ component,

$$
\begin{aligned}
\partial_{4}{ }^{2} \tilde{\Phi}+ & \tilde{\Phi}^{\prime \prime}+\frac{\alpha^{\prime}}{\alpha} \tilde{\Phi}^{\prime}+\frac{\alpha^{\prime}}{2 \alpha} h_{4}^{\prime}= \\
& \frac{1}{2 M_{6}^{4}}\left(\tau^{a}{ }_{a}-4 \tau_{\phi}^{\phi}-\frac{3 M_{5}^{3}}{2} \partial_{4}{ }^{2} \tilde{\Phi}+7 \lambda_{5} \tilde{\Phi}-\frac{M_{5}^{3}}{2}\left(\partial^{\lambda} \partial_{\sigma} h_{\lambda}^{\sigma}-\partial_{4}{ }^{2} h_{4}\right)\right) \delta\left(\rho-r_{0}\right),
\end{aligned}
$$

and finally the $4 D$ tensor equations,

$$
\begin{aligned}
& \partial^{\mu} \partial_{\lambda} h^{\lambda}{ }_{\nu}+\partial_{\nu} \partial_{\lambda} h^{\mu \lambda}-\nabla_{6}{ }^{2} h^{\mu}{ }_{\nu}-\partial^{\mu} \partial_{\nu} h_{4}-\partial^{\mu} \partial_{\nu} \tilde{\Phi}= \\
& \quad \frac{2}{M_{6}^{4}}\left[\tau^{\mu}{ }_{\nu}-\frac{1}{4} \tau^{a}{ }_{a} \delta^{\mu}{ }_{\nu}+\frac{M_{5}^{3}}{2}\left(\partial^{\mu} \partial_{\nu} \tilde{\Phi}-\frac{1}{4} \partial_{4}{ }^{2} \tilde{\Phi} \delta^{\mu}{ }_{\nu}\right)+\frac{\lambda_{5}}{4} \tilde{\Phi} \delta^{\mu}{ }_{\nu}-\right. \\
& \left.\quad \frac{M_{5}^{3}}{2}\left(\partial^{\mu} \partial_{\lambda} h^{\lambda}{ }_{\nu}+\partial_{\nu} \partial_{\lambda} h^{\mu \lambda}-\partial_{4}{ }^{2} h^{\mu}{ }_{\nu}-\partial^{\mu} \partial_{\nu} h_{4}-\frac{1}{4} \delta^{\mu}{ }_{\nu}\left(\partial^{\lambda} \partial_{\sigma} h^{\sigma}{ }_{\lambda}-\partial_{4}{ }^{2} h_{4}\right)\right)\right] \delta\left(\rho-r_{0}\right) .
\end{aligned}
$$

Here $h_{4}=h_{\mu}^{\mu}$ is the trace of the $4 D$ perturbation $h_{\mu \nu}$, the sources $\tau^{a}{ }_{b}$ describing the brane matter perturbations naturally decompose as $\tau^{\mu}{ }_{\nu}$ and $\tau^{\phi}{ }_{\phi}$, and $\tau^{a}{ }_{a}=\tau^{\mu}{ }_{\mu}+\tau_{\phi}^{\phi}$ the full $5 D$ trace of $\tau_{b}^{a}$. The covariant d'Alembertian $\nabla_{6}^{2}$ is defined as before, with respect to the full background metric (25). 
This system contains redundancies, which we need to disentangle away. First, we need to break down the perturbation $h^{\mu}{ }_{\nu}$ into irreducible $4 D$ representations of the Lorentz group, which is the symmetry of the background (25). Using the flat limit of the decomposition theorem in [25], we can write

$$
h^{\mu}{ }_{\nu}=\gamma_{\nu}^{\mu}+\partial^{\mu} A_{\nu}+\partial_{\nu} A^{\mu}+\partial^{\mu} \partial_{\nu} \Psi-\frac{1}{4} \delta^{\mu}{ }_{\nu} \partial_{4}{ }^{2} \Psi+\frac{1}{4} \delta^{\mu}{ }_{\nu} h_{4},
$$

where $\gamma^{\mu}{ }_{\nu}$ is a transverse-traceless (TT) tensor satisfying $\partial_{\mu} \gamma^{\mu}{ }_{\nu}=\gamma^{\mu}{ }_{\mu}=0$, with 5 helicities, $A_{\mu}$ is a Lorenz-gauge vector, $\partial_{\mu} A^{\mu}=0$, with 3 helicities, and $\Psi$ and $h_{4}=h^{\mu}{ }_{\mu}$ are two scalars. The total number of degrees of freedom adds up to ten, which is the number of independent components of a symmetric $4 \times 4$ tensor. Once we recall that these modes are all dependent on $\rho$ and, in principle, could also depend on $\phi$, we really end up with KK towers of degrees of freedom classified by their $4 D$ masses in addition to their helicities. Of course, not all of these modes may end up being physical; some could be pure gauge modes, and one has to carefully check that using the field equations and the gauge symmetries of the problem. As we will only work with axially symmetric perturbations, our task will be considerably simpler.

The field equations (70)-(73) for the linearized theory also decompose by helicities due to $4 D$ Lorentz invariance of the background. A simple way to separate out the equations is to define the transverse-traceless tensor projection operator $K_{\mu \nu \alpha \beta}$, such that $K_{\mu \nu}{ }^{\alpha \beta} h_{\alpha \beta}=\gamma_{\mu \nu}$. This operator must satisfy $K_{\mu \nu \alpha \beta}=K_{\nu \mu \alpha \beta}=K_{\mu \nu \beta \alpha}$ and $K_{\mu \alpha \beta}^{\mu}=\partial^{\mu} K_{\mu \nu \alpha \beta}=0$. The unique solution is

$$
\begin{aligned}
K_{\mu \nu \alpha \beta}= & -\frac{1}{3}\left[\eta_{\mu \nu} \eta_{\alpha \beta}-\frac{3}{2}\left(\eta_{\mu \alpha} \eta_{\nu \beta}+\eta_{\mu \beta} \eta_{\nu \alpha}\right)-\eta_{\mu \nu} \frac{\partial_{\alpha} \partial_{\beta}}{\partial_{4}{ }^{2}}-\eta_{\alpha \beta} \frac{\partial_{\mu} \partial_{\nu}}{\partial_{4}{ }^{2}}\right. \\
& \left.+\frac{3}{2}\left(\eta_{\nu \beta} \frac{\partial_{\mu} \partial_{\alpha}}{\partial_{4}{ }^{2}}+\eta_{\mu \beta} \frac{\partial_{\nu} \partial_{\alpha}}{\partial_{4}{ }^{2}}+\eta_{\nu \alpha} \frac{\partial_{\mu} \partial_{\beta}}{\partial_{4}{ }^{2}}+\eta_{\mu \alpha} \frac{\partial_{\nu} \partial_{\beta}}{\partial_{4}{ }^{2}}\right)-2 \frac{\partial_{\mu} \partial_{\nu} \partial_{\alpha} \partial_{\beta}}{\partial_{4}{ }^{2}}\right] .
\end{aligned}
$$

Clearly, this operator annihilates the vector and scalar terms in (74), and when we apply it to Eq. (73), it projects out the TT contributions. Since the vacuum (25) is $4 D$-flat, stress energy conservation implies $\partial_{\mu} \tau^{\mu}{ }_{\nu}=0$, and so

$$
K_{\mu \nu}{ }^{\alpha \beta} \tau_{\alpha \beta}=\tau_{\nu}^{\mu}-\frac{1}{3}\left(\delta^{\mu}{ }_{\nu}-\frac{\partial^{\mu} \partial_{\nu}}{\partial_{4}{ }^{2}}\right) \tau_{\alpha}^{\alpha}
$$

Hence our final form of the TT-tensor field equation is

$$
\nabla_{6}{ }^{2} \gamma_{\nu}^{\mu}+\frac{M_{5}^{3}}{M_{6}^{4}} \partial_{4}{ }^{2} \gamma^{\mu}{ }_{\nu} \delta\left(\rho-r_{0}\right)=-\frac{2}{M_{6}^{4}}\left[\tau^{\mu}{ }_{\nu}-\frac{1}{3}\left(\delta_{\nu}^{\mu}-\frac{\partial^{\mu} \partial_{\nu}}{\partial_{4}{ }^{2}}\right) \tau^{\alpha}{ }_{\alpha}\right] \delta\left(\rho-r_{0}\right) .
$$

Subtracting the TT-tensor equation from (73) leads to the system which contains only the vector and scalar contributions. We can extract the vector in a similar way as the TT-tensor, by defining the vector projection operator $V_{\alpha \beta}$, and acting on the momentum constraint (70). Since the backgrounds (25) are Lorentz-invariant, in the linear order of perturbation theory vector sources vanish and the vector modes decouple from the matter distribution. Hence we can set the vector field in (74) to zero. 
This leaves us with scalars. In brane-fixed Gaussian-normal gauge, they are $\tilde{\Phi}, \Psi$ and $h_{4}$, obeying the field equations (70)-(72) and what remains of (73) after we subtract out (77). To pick out the minimal set of independent equations for scalars from the system (70)-(73), we define $X=\frac{1}{4}\left(h_{4}-\partial_{4}{ }^{2} \Psi\right)$, write the scalar part of the metric perturbation as

$$
{ }^{s} h^{\mu}{ }_{\nu}=\partial^{\mu} \partial_{\nu} \Psi+X \delta^{\mu}{ }_{\nu},
$$

and substitute it into the difference of Eqs. (73) and (77). The result is a combination of two independent $4 D$ tensors, diagonal $\propto \delta^{\mu}{ }_{\nu}$ and off-diagonal $\propto \partial^{\mu} \partial_{\nu}$. Hence the coefficients of these tensors must separately vanish. Likewise, we could write the $4 D$ trace of this equation. However it is a trivial linear combination of the diagonal and off-diagonal terms, and so we can ignore it immediately. Then, combining these equations with (70)-(72), and substituting (78) for $h_{\mu \nu}$, we get

$$
\begin{aligned}
& \partial_{\lambda}\left(3 X^{\prime}+\tilde{\Phi}^{\prime}+\frac{\alpha^{\prime}}{2 \alpha} \tilde{\Phi}\right)=0 \\
& \tilde{\Phi}^{\prime \prime}+\frac{\alpha^{\prime}}{\alpha} \tilde{\Phi}^{\prime}+\partial_{4}{ }^{2} \Psi^{\prime \prime}+4 X^{\prime \prime}=\frac{1}{2 M_{6}^{4}}\left(\tau^{\mu}{ }_{\mu}+\tau_{\phi}^{\phi}+3 \lambda_{5} \tilde{\Phi}-\frac{3 M_{5}^{3}}{2} \partial_{4}{ }^{2}(\tilde{\Phi}+3 X)\right) \delta\left(\rho-r_{0}\right), \\
& \partial_{4}{ }^{2} \tilde{\Phi}+\tilde{\Phi}^{\prime \prime}+\frac{\alpha^{\prime}}{\alpha} \tilde{\Phi}^{\prime}+\frac{\alpha^{\prime}}{2 \alpha}\left(\partial_{4}{ }^{2} \Psi^{\prime}+4 X^{\prime}\right)= \\
& =\frac{1}{2 M_{6}^{4}}\left(\tau^{\mu}{ }_{\mu}-3 \tau_{\phi}^{\phi}+7 \lambda_{5} \tilde{\Phi}-\frac{3 M_{5}^{3}}{2} \partial_{4}{ }^{2}(\tilde{\Phi}-X)\right) \delta\left(\rho-r_{0}\right), \\
& \Psi^{\prime \prime}+\frac{\alpha^{\prime}}{2 \alpha} \Psi^{\prime}+\tilde{\Phi}+2 X=\frac{1}{M_{6}^{4}}\left(\frac{2}{3 M_{5}^{3}} \partial_{4}{ }^{-2}\left(\tau^{\mu}{ }_{\mu}\right)-M_{5}^{3}(\tilde{\Phi}+2 X)\right) \delta\left(\rho-r_{0}\right), \\
& X^{\prime \prime}+\frac{\alpha^{\prime}}{2 \alpha} X^{\prime}+\partial_{4}{ }^{2} X=-\frac{1}{4 M_{6}^{4}}\left(\frac{2}{3}\left(\tau_{\mu}^{\mu}-3 \tau_{\phi}^{\phi}\right)+2 \lambda_{5} \tilde{\Phi}-M_{5}^{3} \partial_{4}{ }^{2}(\tilde{\Phi}-X)\right) \delta\left(\rho-r_{0}\right),
\end{aligned}
$$

where we have decomposed the full d'Alembertian as $\nabla_{6}{ }^{2}=\partial_{4}{ }^{2}+\nabla_{\vec{y}}^{2}$ and used the formula for axially symmetric fields $\nabla_{\vec{y}}^{2}=\frac{d^{2}}{d \rho^{2}}+\frac{\alpha^{\prime}}{2 \alpha} \frac{d}{d \rho}$. We have replaced the $5 D$ stress-energy trace by $\tau^{a}{ }_{a}=\tau_{\mu}^{\mu}+\tau_{\phi}^{\phi}$. In Eq. (79), we can immediately omit the $4 D$ divergence $\partial_{\lambda}$ because the $4 D$ background is flat Minkowski. In Eq. (82), $\partial_{4}{ }^{-2}$ acting on $\tau^{\mu}{ }_{\mu}$ can be simply understood as a multiplication of the Fourier components of $\tau^{\mu}{ }_{\mu}$ by $-1 / k^{2}$, as we will do later on. Finally, we note that the bulk terms in Eq. (83) depend only on $X$, and hence can be readily integrated. Thus we should keep it in our final set of independent field equations.

We find that if we add Eqs. (80) and (81), and subtract from the result $\partial_{4}{ }^{2} \times$ Eq. (82) and $4 \times$ Eq. (83), the result is a linear combination of the $\rho$-derivative of Eq. (79) and Eq. (83). Next, we can similarly start with Eq. (80) but now subtract Eq. (81), $\partial_{4}{ }^{2} \times$ Eq. (82) and $4 \times$ Eq. (83). The result is very simple:

$$
\partial_{4}^{2}(\tilde{\Phi}+3 X)+\frac{\alpha^{\prime}}{2 \alpha}\left(\partial_{4}{ }^{2} \Psi^{\prime}+4 X^{\prime}\right)=0 .
$$

In this equation, the sources on the right hand-side completely cancel, since they are localized on the brane. The reason is that this is the perturbed gravitational equation in the radial direction, which is sourceless in brane-fixed Gaussian-normal gauge. This equation is 
particularly useful when we look for the solutions in the bulk, as we will see below. Further, we can also check, by using the $\rho$-derivative of (79), that the bulk part of Eq. (82) reduces precisely to Eq. (84).

These observations teach us that in the bulk we can trade Eqs. (80), (81) and (82) for one independent combination, which, with wisdom after the fact, we choose to be precisely Eq. (84). This, however, is slightly tricky on the brane itself. A product of functions which are discontinuous on the brane appears in Eq. (84): $\frac{\alpha^{\prime}}{\alpha}$ and $\partial_{4}{ }^{2} \Psi^{\prime}+4 X^{\prime}$. Hence although this equation contains no terms $\propto \delta\left(\rho-r_{0}\right)$, it still gives some information about the boundary

behavior of the scalars, albeit weaker. It requires that $\Delta\left(\frac{\alpha^{\prime}}{2 \alpha}\left(\partial_{4}{ }^{2} \Psi^{\prime}+4 X^{\prime}\right)\right)=0$. Although the factors are bounded, because they are discontinuous this condition - by itself - is degenerate: it relates the jumps of the functions to their values on different sides of the brane. So using it alone we can't extract unambiguously the junction conditions for $\partial_{4}{ }^{2} \Psi^{\prime}+4 X^{\prime}$. However, the degeneracies in the boundary information in (84) are completely artificial. They are easily lifted by going back to any of the boundary conditions encoded in the $\delta$-function terms in either of Eqs. (80), (81) and (82). From examining these equations it is clear that the most useful form of the boundary condition is obtained from Eq. (82), because the boundary condition for $\tilde{\Phi}$ is tied to the one for $X$ by Eq. (79). Thus, our independent equations in the bulk are (79), (83) and (84), supplemented with the boundary conditions obtained by Gaussian pillbox integration of (82) and (83) around the brane. Further, we can immediately integrate (79) once, with respect to $x^{\lambda}$, and ignore the non-normalizable homogeneous mode on the brane. With this, finally, our system of equations reduces to the bulk system

$$
\begin{aligned}
& 3 X^{\prime}+\tilde{\Phi}^{\prime}+\frac{\alpha^{\prime}}{2 \alpha} \tilde{\Phi}=0 \\
& \partial_{4}^{2}(\tilde{\Phi}+3 X)+\frac{\alpha^{\prime}}{2 \alpha}\left(\partial_{4}{ }^{2} \Psi^{\prime}+4 X^{\prime}\right)=0, \\
& X^{\prime \prime}+\frac{\alpha^{\prime}}{2 \alpha} X^{\prime}+\partial_{4}^{2} X=0, \\
& \Delta X^{\prime}=-\frac{1}{4 M_{6}^{4}}\left(\frac{2}{3}\left(\tau_{\mu}^{\mu}-3 \tau_{\phi}^{\phi}\right)+2 \lambda_{5} \tilde{\Phi}-M_{5}^{3} \partial_{4}{ }^{2}(\tilde{\Phi}-X)\right), \\
& \Delta \Psi^{\prime}=-\frac{M_{5}^{3}}{M_{6}^{4}}(\tilde{\Phi}+2 X),
\end{aligned}
$$

where the last two encode the boundary conditions, equating the jumps of bulk derivatives to the brane stress-energy. This set of equations is fairly straightforward to solve: Eq. (85) relates $\tilde{\Phi}$ to $X$, which is determined by Eq. (87) and boundary condition (88). Then Eqs. (86) and (89) determine the remaining variable, $\Psi$.

\subsection{Solutions}

We now turn to finding the solutions of the system (77), (85)-(89). The most interesting solutions are those which describe the long range fields of static sources on the brane. They are our key probe of what kind of gravity the theory reduces to at long distances. One could quite straightforwardly also determine the spectrum of the theory around the vacuum, when the stress-energy sources $\tau_{b}^{a}=0$. We have done it, to find that the spectrum contains 
a continuum of massive tensors and a scalar, fully described by one independent variable $X$. In this section we will give the solutions of linearized theory which apply to a general case of arbitrary time dependent solutions. However what is really important is to see how these modes couple to matter in order to determine just how dangerous, or not, the extra modes may be at long distances. For this reason, we will focus on the long range static fields, completely determined by the static rings of matter on the brane. We will also write down the formal solution for the full metric perturbation $h^{\mu}{ }_{\nu}$, which will enable us to see the tensor structure of full long range gravity in the linearized theory.

Let us start with the field equation governing the tensor modes, Eq. (77). This equation can be solved very easily using the standard Green's function techniques. Expanding out the bulk d'Alembertian $\nabla_{6}{ }^{2}$, and Fourier-transforming over the $4 D$ variables $x^{\mu}$, while keeping in mind that the fields are axially symmetric, to $\gamma^{\mu}{ }_{\nu}(x, \rho)=\int \frac{d^{4} k}{(2 \pi)^{4}} \gamma^{\mu}{ }_{\nu}(k, \rho) \exp (i k \cdot x)$ and $\tau_{b}^{a}(x, \rho)=\int \frac{d^{4} k}{(2 \pi)^{4}} \tau_{b}^{a}(k, \rho) \exp (i k \cdot x)$, we can rewrite the TT-tensor equation as

$$
\gamma_{\nu}^{\mu}{ }_{\nu}^{\prime \prime}+\frac{\alpha^{\prime}}{2 \alpha} \gamma^{\mu}{ }_{\nu}{ }^{\prime}-k^{2} \gamma^{\mu}{ }_{\nu}-\frac{M_{5}^{3}}{M_{6}^{4}} k^{2} \gamma^{\mu}{ }_{\nu} \delta\left(\rho-r_{0}\right)=-\frac{2}{M_{6}^{4}}\left[\tau^{\mu}{ }_{\nu}-\frac{1}{3}\left(\delta^{\mu}{ }_{\nu}-\frac{k^{\mu} k_{\nu}}{k^{2}}\right) \tau^{\alpha}{ }_{\alpha}\right] \delta\left(\rho-r_{0}\right)
$$

The solution of this equation can be written in terms of the Green's function $\mathcal{G}_{\mathrm{TT}}(k, \rho)$, defined by the equation

$$
\mathcal{G}_{\mathrm{TT}}^{\prime \prime}+\frac{\alpha^{\prime}}{2 \alpha} \mathcal{G}_{\mathrm{TT}}{ }^{\prime}-k^{2} \mathcal{G}_{\mathrm{TT}}-\frac{M_{5}^{3}}{M_{6}^{4}} k^{2} \mathcal{G}_{\mathrm{TT}} \delta\left(\rho-r_{0}\right)=\frac{1}{M_{6}^{4}} \delta\left(\rho-r_{0}\right) .
$$

Then the solution is simply

$$
\gamma_{\nu}^{\mu}(k, \rho)=-2 \mathcal{G}_{\text {TT }}(k, \rho)\left[\tau^{\mu}{ }_{\nu}-\frac{1}{3}\left(\delta^{\mu}{ }_{\nu}-\frac{k^{\mu} k_{\nu}}{k^{2}}\right) \tau^{\alpha}{ }_{\alpha}\right]
$$

where we postpone the detailed determination of $\mathcal{G}_{\text {TT }}$ until later. For now, it is enough to see that it exists, after we specify appropriate boundary conditions at bulk infinity and inside the wrapped brane, which ensure the regularity of the solution.

The scalar story is more intricate. The reason is that the backgrounds (25), which we take as $4 D$ vacua of the theory, are not vacuum solutions from either the bulk or the wrapped 4-brane theory points of view, because they contain a non-trivial $\Sigma$ field contribution to stress-energy on the brane. As we discussed, that is necessary to wrap the brane on a circle and make it look like a codimension- 2 object. However, because of this, the brane bending term will always appear in perturbation theory around the wrapped vacuum, even if the matter perturbations $\tau_{b}^{a}$ vanish, in contrast to the simpler codimension-1 cases studied in [67]. This is interesting because it lends to a difference in the couplings of scalar metric perturbations, and the helicity-0 modes from the massive graviton multiplets at the same mass levels.

So let us construct the solution of (85)-(89). We first Fourier-transform all the fields over the $4 D x^{\mu}$ space, as in the case of TT-tensors above. Then we take a slightly roundabout approach, due to the presence of the background flux of $\Sigma$, reflected by the tension-dependent terms in (85)-(89) (explicit, as well as implicit in $\frac{\alpha^{\prime}}{\alpha}$ ). We need to carefully identify how they mix different scalar fields. To this end it is useful to recall the gauge transformations (68), 
and explicitly restore the brane bending term $\xi$ which keeps the brane-localized terms in check. Using (68) and the background equation $\left(\frac{\alpha^{\prime}}{\alpha}\right)^{\prime}=-\frac{\alpha^{\prime 2}}{2 \alpha^{2}}-4 \frac{\lambda_{5}}{M_{6}^{4}} \delta\left(\rho-r_{0}\right)$, we can check that $\tilde{\Phi}^{\prime}+\frac{\alpha^{\prime}}{2 \alpha} \tilde{\Phi}=\Phi^{\prime}$. Substituting this in Eq. (85), we find $3 X^{\prime}+\Phi^{\prime}=4 \frac{\lambda_{5}}{M_{6}^{4}} \xi \delta\left(\rho-r_{0}\right)$, which is very easy to integrate over $\rho$ : we get $3 X+\Phi=4 \frac{\lambda_{5}}{M_{6}^{4}} \xi \Theta(y)+F$, where $F$ is a Fourier-transform of some function $F(x)$. However, we recall that this equation was already integrated once over $x^{\mu}$, which implies that $\partial_{\mu} F=0$ for consistency, or $F=$ const.. However, any such integration constant can be readily removed by a residual $4 D$ diffeomorphism of the form $\chi^{\mu}=\frac{F}{2} x^{\mu}$ as one can immediately check. Thus we can set $F=0$, to find

$$
\Phi=-3 X+\frac{4 \lambda_{5}}{M_{6}^{4}} \xi \Theta\left(\rho-r_{0}\right)
$$

Again using (68) and noting the integral identity $\int_{r_{0}}^{\rho} d \rho \Theta\left(\rho-r_{0}\right)=\left(\rho-r_{0}\right) \Theta\left(\rho-r_{0}\right)$, we can write the solution for $\tilde{\Phi}$ :

$$
\tilde{\Phi}=-3 X+\frac{3 \alpha^{\prime}}{2 \alpha} \int_{r_{0}}^{\rho} d \rho X+\frac{\alpha^{\prime}}{\alpha} \xi+\frac{4 \lambda_{5}}{M_{6}^{4}} \xi \Theta\left(\rho-r_{0}\right)\left[1-\frac{\alpha^{\prime}}{2 \alpha}\left(\rho-r_{0}\right)\right] .
$$

In particular, on the brane it is given by $\tilde{\Phi}\left(r_{0}\right)=-3 X\left(r_{0}\right)+\frac{2}{r_{0}} \xi$.

Next we solve for $\Psi$, by substituting Eq. (94) into Eq. (86), and defining

$$
\Upsilon^{\prime}=\Psi^{\prime}+3 \int_{r_{0}}^{\rho} d \rho X+2 \xi-4 \frac{\lambda_{5}}{M_{6}^{4}} \xi \Theta\left(\rho-r_{0}\right)
$$

With this, Eq. (86) becomes

$$
\frac{\alpha^{\prime}}{2 \alpha}\left(\Upsilon^{\prime}-\frac{4 X^{\prime}}{k^{2}}\right)=-4 \frac{\lambda_{5}}{M_{6}^{4}} \xi \Theta\left(\rho-r_{0}\right)
$$

These two equations are straightforward to integrate. After a simple algebra we find

$$
\Psi=\frac{4}{k^{2}} X-3 \int_{r_{0}}^{\rho} d \rho \int_{r_{0}}^{\rho} d \rho X(\rho)-2 \rho \xi+\frac{2 \lambda_{5}}{M_{6}^{4}} \xi \int_{r_{0}}^{\rho} \frac{d \rho}{\left(\frac{\alpha^{\prime}}{\alpha}\right)} \Theta\left(\rho-r_{0}\right)\left(2\left(\rho-r_{0}\right) \frac{\alpha^{\prime}}{\alpha}-4\right),
$$

where we have subtracted, using another residual $4 D$ diffeomorphism, an arbitrary $4 D$ wave which arises as the Fourier transform of the constants of integrations that yield (93). On the brane, this yields $\Psi=\frac{4}{k^{2}} X-2 r_{0} \xi$. Before proceeding, note that both solutions for $\tilde{\Phi}$ and $\Psi$, Eqs. (94) and (97), remain indifferent to the presence of brane matter sources, which are completely encoded in the bulk field $X$ and the brane bending $\xi$. Once we find solutions for $X$ and $\xi$ in the presence of sources, we can just substitute them back into (94) and (97) and have $\tilde{\Phi}$ and $\Psi$ as well. This greatly facilitates integration, and is the reason behind our choice of Eqs. (85)-(89) for the description of the scalar sector.

So let us determine $X$ and $\xi$. First, we solve for $\xi$ using the boundary conditions (88) and (89) and solutions (94) and (97). Using the expression for $\tilde{\Phi}$ on the brane given above we can eliminate $\tilde{\Phi}$ from the boundary conditions. Then we use the solution (89) to relate $\Delta \Psi^{\prime}$ to $\Delta X^{\prime}$, which gives

$$
\Delta \Psi^{\prime}=\frac{4}{k^{2}} \Delta X^{\prime}-\frac{8 \lambda_{5}}{\left.M_{6}^{4}\left(\frac{\alpha^{\prime}}{\alpha}\right)\right|_{+}} \xi .
$$


Finally, using (88) to evaluate $\Delta X^{\prime}$, we compare (89) and (98), and compute $\xi$ in terms of the value of $X$ on the brane. A simple albeit tedious algebra yields

$$
\xi=\frac{3(1-b) M_{5}^{3} r_{0}^{2}}{2 b M_{6}^{4}} \frac{k^{2}+\frac{b M_{6}^{4}}{M_{5}^{3} r_{0}}}{1-b+k^{2} r_{0}^{2}} X+\frac{(1-b) r_{0}^{2}}{b M_{6}^{4}} \frac{\tau_{\phi}^{\phi}}{1-b+k^{2} r_{0}^{2}},
$$

where we have used $\left.\left(\frac{\alpha^{\prime}}{\alpha}\right)\right|_{+}=\frac{2}{r_{0}}-\frac{4 \lambda_{5}}{M_{6}^{4}}$, obtained from (62), and resorted to Eq. (23) to reintroduce $b=\frac{2 \lambda_{5} r_{0}}{M_{6}^{4}}$. We notice, that in the thin brane limit, $r_{0} \rightarrow 0$, the brane bending term formally vanishes, if we hold all other terms in Eq. (99) fixed. Now we can finally go after $X$. Using the expressions for $\tilde{\Phi}=-3 X\left(r_{0}\right)+\frac{2}{r_{0}} \xi$ on the brane, and with $\xi$ given in Eq. (99), we can eliminate them from the boundary condition (88) in favor of $X$. This yields

$$
\Delta X^{\prime}=\frac{M_{5}^{3}}{M_{6}^{4}}\left(k^{2}+\frac{3 b M_{6}^{4}}{4 M_{5}^{3} r_{0}}-\beta\left(k^{2}\right)\right) X-\frac{1}{6 M_{6}^{4}}\left(\tau_{\alpha}^{\alpha}+\frac{3 M_{5}^{3} r_{0} k^{2}}{b M_{6}^{4}} \frac{1-b-\frac{b M_{6}^{4} r_{0}}{M_{5}^{3}}}{1-b+k^{2} r_{0}^{2}} \tau_{\phi}^{\phi}\right),
$$

where

$$
\beta\left(k^{2}\right)=\frac{3 M_{5}^{3} r_{0}}{4 b M_{6}^{4}} \frac{1-b}{1-b+k^{2} r_{0}^{2}}\left(k^{2}+\frac{b M_{6}^{4}}{M_{5}^{3} r_{0}}\right)^{2},
$$

is the correction to the brane kinetic term of the scalar $X$ originating from the brane bending contributions. The field equation in the bulk (87) is very simple. If we rewrite it with the boundary condition (100) restored as a $\delta$-function term, in the Schrödinger form, we can compare it to the TT-tensor field equations (90). The equation is

$$
\begin{aligned}
X^{\prime \prime}+\frac{\alpha^{\prime}}{2 \alpha} X^{\prime}-k^{2} X-\frac{M_{5}^{3}}{M_{6}^{4}} & \left(k^{2}+\frac{3 b M_{6}^{4}}{4 M_{5}^{3} r_{0}}-\beta\left(k^{2}\right)\right) X \delta\left(\rho-r_{0}\right)= \\
& -\frac{1}{6 M_{6}^{4}}\left(\tau^{\alpha}{ }_{\alpha}+\frac{3 M_{5}^{3} r_{0} k^{2}}{b M_{6}^{4}} \frac{1-b-\frac{b M_{6}^{4} r_{0}}{M_{5}^{3}}}{1-b+k^{2} r_{0}^{2}} \tau_{\phi}^{\phi}\right) \delta\left(\rho-r_{0}\right) .
\end{aligned}
$$

This equation is very similar to the TT-tensor equation (90), with the differences being in the brane-localized terms. We will comment on them below. Here, we note that we can now exploit the formal similarity with TT-tensor equation and use the Green's function methods again, to find the solution. This time we define the scalar Green's function $\mathcal{G}_{\mathrm{x}}(k, \rho)$ by

$$
\mathcal{G}_{\mathrm{X}}{ }^{\prime \prime}+\frac{\alpha^{\prime}}{2 \alpha} \mathcal{G}_{\mathrm{x}}{ }^{\prime}-k^{2} \mathcal{G}_{\mathrm{X}}-\frac{M_{5}^{3}}{M_{6}^{4}}\left(k^{2}+\frac{3 b M_{6}^{4}}{4 M_{5}^{3} r_{0}}-\beta\left(k^{2}\right)\right) \mathcal{G}_{\mathrm{X}} \delta\left(\rho-r_{0}\right)=\frac{1}{M_{6}^{4}} \delta\left(\rho-r_{0}\right)
$$

Formally the solution is

$$
X(k, \rho)=-\frac{1}{6} \mathcal{G}_{\mathrm{X}}(k, \rho)\left[\tau^{\alpha}{ }_{\alpha}+\frac{3 M_{5}^{3} r_{0} k^{2}}{b M_{6}^{4}} \frac{1-b-\frac{b M_{6}^{4} r_{0}}{M_{5}^{3}}}{1-b+k^{2} r_{0}^{2}} \tau_{\phi}^{\phi}\right]
$$

where again we defer the detailed determination of $\mathcal{G}_{\mathrm{x}}$ until later.

Let us now briefly outline here the procedure for extracting the long range physical fields from the solutions. We recall that by axial symmetry of the configurations which we are 
exploring, at distances $\ell>r_{0}$, we can dimensionally reduce the theory on the compact circle. Hence the long distance dynamics will really be described by a scalar-tensor theory, where the Brans-Dicke-like scalar field, or the radion, is $g_{\phi \phi}^{1 / 2}$. In addition, in the tensor sector there will be the helicity-0 mode resonance as well as the helicity-2 ones. The radion $g_{\phi \phi}^{1 / 2}$ already renders the effective gravitational strength as evaluated from the brane-induced gravity action field-dependent. Indeed, at the linearized level about the vacuum, we see from Eq. (69) that since we are working in Gaussian-normal brane-fixed gauge, the scalar field will be $\chi_{B D}=\left(1+\frac{\tilde{\Phi}}{2}\right) \alpha^{1 / 2}(\rho)$, where $\alpha^{1 / 2}(\rho)$ is its background value, and $\alpha^{1 / 2}(\rho) \frac{\tilde{\Phi}}{2}$ the perturbation sourced by the rings of matter on the wrapped brane. Clearly, the background variation of $\chi_{B D}$ retains the memory of the compactified dimension, which opens up as one moves away from the cylindrical brane, as discussed recently in [59]. Nevertheless, the description in terms of the reduced theory is still perfectly justified at the linearized level with axisymmetric sources. It is then useful to go to the analogue of the effective 'Einstein' frame for the dimensionally reduced perturbation theory, in order to separate out graviton and scalar forces, and compare the linearized fields to $4 D$ General Relativity. In doing so, we will ignore the prefactors $\alpha^{1 / 2}$ as they are fully absorbed in the background solution, and only remove the perturbations $\tilde{\Phi}$ from the effective Planck scales, because we are most interested in the effective theory along the brane. Thus the radial variation of the effective bulk Planck scale is not important at the linearized level of perturbation theory. So we proceed by noting that the graviton kinetic terms in the full $6 D$ theory come from

$$
S_{6 D} \ni \int d^{6} x \sqrt{g_{6}} \frac{M_{6}^{4}}{2} R_{6}+\int_{\rho=r_{0}} d^{5} x \sqrt{g_{5}} \frac{M_{5}^{3}}{2} R_{5}
$$

Then dimensionally reducing on the circle changes these terms to

$$
S_{6 D} \ni \int d^{5} x \sqrt{g_{5}} \pi M_{6}^{4} \alpha^{1 / 2}(\rho)\left(1+\frac{\tilde{\Phi}}{2}\right) R_{5}+\int_{\rho=r_{0}} d^{4} x \sqrt{g_{4}} \pi M_{5}^{3} r_{0}\left(1+\frac{\tilde{\Phi}}{2}\right) R_{4} .
$$

To go to the effective 'Einstein' frame, we absorb the radion perturbation by conformally transforming the metric to $g_{A B}^{\mathrm{E}}=\Omega^{2} g_{A B}$. Although the graviton kinetic terms live in different dimensions, we can render the conformal rescalings of the two terms the same thanks to the fact that we are working entirely in the Gaussian-normal brane-fixed gauge. Since our starting metric satisfies $g_{\rho \rho}=1, g_{\rho \mu}=0$, we can change the radial coordinate to $\bar{\rho}=\int d \rho \Omega$ to ensure that the bulk term changes under the conformal transformation as $d \rho \sqrt{g_{5}} R_{5}=d \rho^{\prime} \sqrt{g_{5}^{\mathrm{E}}} R_{5}^{\mathrm{E}} / \Omega^{2}$, i.e. the same as the brane term. We bear in mind that we will need to change $x^{\mu}$ coordinates in order to keep the reduced metric in Gaussiannormal brane fixed gauge. So to absorb the factors $\propto \tilde{\Phi}$ from in front of the graviton kinetic terms (106) we need $\Omega^{2}=1+\frac{\tilde{\Phi}}{2}$. This defines our radial gauge transformation by $\bar{\rho}=\int_{r_{0}}^{\rho} d \rho\left(1+\frac{\tilde{\Phi}}{4}\right)+r_{0}=\rho+\frac{1}{4} \int_{r_{0}}^{\rho} d \rho \tilde{\Phi}$ where we have chosen the integration constant to ensure that the brane remains fixed, at $\bar{\rho}=r_{0}$. This transformation is of the form of general diffeomorphisms (61) with $\chi^{\rho}=\frac{1}{4} \int_{r_{0}}^{\rho} d \rho \tilde{\Phi}$. Then to keep the perturbation $\bar{h}_{\mu \rho}$ from appearing and ensure that we are still in the Gaussian-normal gauge, we need to pick $\chi_{\mu}^{\prime}=-\partial_{\mu} \chi^{\rho}$, or therefore $\chi_{\mu}=-\frac{1}{4} \int_{r_{0}}^{\rho} d \rho \int_{r_{0}}^{\rho} d \rho \partial_{\mu} \tilde{\Phi}$ (where we can ignore the overbars in these expressions to the leading order). 
But now, from these expressions we immediately see that the gauge transformations vanish on the brane! Hence, the gauge fixing on the brane is unaffected by the dimensional reduction, and when calculating the fields along the brane we can just use the expressions in the original coordinates, ignoring altogether their behavior in the bulk ${ }^{2}$. Thus we can use the conformal transformation $g_{\mu \nu}^{\mathrm{E}}=\left(1+\frac{\Phi}{2}\right) g_{\mu \nu}$ on the brane. Now after Fourier transforming the decomposition theorem formula (74), and replacing $h_{4}$ by $X$ using Eq. (78), the full metric perturbation along the brane in the original frame is

$$
h^{\mu}{ }_{\nu}=\gamma_{\nu}^{\mu}-k^{\mu} k_{\nu} \Psi+X \delta^{\mu}{ }_{\nu}
$$

where on the brane $\Psi=\frac{4}{k^{2}} X-2 r_{0} \xi$. After our conformal transformation to linear order in perturbation, we find that $h_{\mu \nu}^{\mathrm{E}}=h_{\mu \nu}+\frac{\tilde{\Phi}}{2} \eta_{\mu \nu}$, or therefore, using the expressions for $\tilde{\Phi}, \Psi$ on the brane to express them all in terms of the variables $X$ and $\xi$,

$$
h_{\nu}^{\mathrm{E} \mu}=\gamma_{\nu}^{\mu}-4\left(X-\frac{r_{0} k^{2}}{2} \xi\right) \frac{k^{\mu} k_{\nu}}{k^{2}}+\left(\frac{\xi}{r_{0}}-\frac{X}{2}\right) \delta_{\nu}^{\mu},
$$

where of course $\xi$ is given by (99). This is the effective 'Einstein' frame field of matter rings on the wrapped brane, which may yet contain extra scalar-like contributions from the helicity-0 modes. To see how it behaves we now need to find out the explicit forms of Green's functions $\mathcal{G}_{\text {TT }}$ and $\mathcal{G}_{\mathrm{X}}$.

\subsection{Fields of Static Sources Along the Brane}

Since we are looking for the static solutions, obeying $k^{\mu}=(0, \vec{k})$, which implies that all the momentum space Fourier transforms are formally $\mathcal{G}(k)=2 \pi \delta\left(k^{0}\right) \mathcal{G}(\vec{k})$, we can factorize out the energy Green's function, set $k^{0}=0$ in the formulas of the previous section, and drop the integration $\int \frac{d k^{0}}{2 \pi}$ from the Fourier integral. We then solve the Green's function equations (91) and (103) using - again, as in the construction of the shock waves - the standard technique of sewing together the solutions of the homogeneous equation on either side of the brane, regular in the center and far away.

Off the brane, both equations (91) and (103) look the same. The solutions which are regular in the center and at infinity are

$$
\mathcal{G}= \begin{cases}A I_{0}(k \rho), & \rho \leq r_{0} \\ B K_{0}\left(k\left(\rho+\frac{b r_{0}}{1-b}\right)\right), & \rho \geq r_{0}\end{cases}
$$

where now $k=|\vec{k}|$. We sew them together at the brane by using the boundary condition found with the Gaussian pillbox integration of Eq. (91), that now gives different results for the two Green's functions,

$$
\Delta \mathcal{G}_{\text {TT }}=\frac{M_{5}^{3}}{M_{6}^{4}} k^{2} \mathcal{G}_{\text {TT }}+\frac{1}{M_{6}^{4}}, \quad \Delta \mathcal{G}_{\mathrm{X}}=\frac{M_{5}^{3}}{M_{6}^{4}}\left[k^{2}+\frac{3 b M_{6}^{4}}{4 M_{5}^{3} r_{0}}-\beta\left(k^{2}\right)\right] \mathcal{G}_{\mathrm{X}}+\frac{1}{M_{6}^{4}},
$$

\footnotetext{
${ }^{2}$ Forearmed with the knowledge that the fields are localized near the brane, which will be ensured by the asymptotic boundary conditions on Green's functions.
} 
with $\beta\left(k^{2}\right)$ given in (101), and then setting the homogeneous solution to zero. This yields

$$
\mathcal{G}_{\mathrm{TT}}=\frac{1}{M_{6}^{4} k} \frac{I_{0}\left(k \rho_{<}\right) K_{0}\left(k \rho_{>}\right)}{I_{0}\left(k r_{0}\right) K_{1}\left(k \frac{r_{0}}{1-b}\right)+I_{1}\left(k r_{0}\right) K_{0}\left(k \frac{r_{0}}{1-b}\right)+\frac{M_{5}^{3} k}{M_{6}^{4}} I_{0}\left(k r_{0}\right) K_{0}\left(k \frac{r_{0}}{1-b}\right)},
$$

and

$$
\mathcal{G}_{\mathrm{x}}=\frac{1}{M_{6}^{4} k} \frac{I_{0}\left(k \rho_{<}\right) K_{0}\left(k \rho_{>}\right)}{I_{0}\left(k r_{0}\right) K_{1}\left(k \frac{r_{0}}{1-b}\right)+I_{1}\left(k r_{0}\right) K_{0}\left(k \frac{r_{0}}{1-b}\right)+\frac{M_{5}^{3}}{M_{6}^{4} k}\left[k^{2}+\frac{3 b M_{6}^{4}}{4 M_{5}^{3} r_{0}}-\beta\left(k^{2}\right)\right] I_{0}\left(k r_{0}\right) K_{0}\left(k \frac{r_{0}}{1-b}\right)},
$$

where, just as in Eq. (47),

$$
I_{0}\left(k \rho_{<}\right) K_{0}\left(k \rho_{>}\right)=\left\{\begin{array}{ll}
I_{0}(k \rho) K_{0}\left(k \frac{r_{0}}{1-b}\right), & \rho \leq r_{0} \\
I_{0}\left(k r_{0}\right) K_{0}\left(k\left(\rho+\frac{b r_{0}}{1-b}\right)\right), & \rho \geq r_{0}
\end{array} .\right.
$$

In fact the Green's function $\mathcal{G}_{\text {Tт }}$ is identical to the Green's function that appears in the shock wave solution (46). This should hardly come as a surprise, because it is obvious from Eq. (90) that it has the same form as the shock wave equation when the stress energy source is traceless. The only difference between these two cases is in the domain of Fourier integration, which for relativistic sources involves one integration fewer because of the Lorenz boost of the longitudinal direction.

Since we wish to explore the fields along the brane, we set $\rho=r_{0}$ in the Green's functions (111) and (112). At distances $\ell \gg r_{0}$, where we can limit our attention to axisymmetric configurations, for which the solutions (111) and (112) are valid, $k r_{0} \sim r_{0} / \ell \ll 1$, we can always replace $I_{0}$ and $I_{1}$ by their small argument expansion, and approximate them by $I_{0} \rightarrow 1, I_{1} \rightarrow 0$. We must be more cautious with $K_{0}$ and $K_{1}$ since they depend on the deficit angle, and for near critical branes $k r_{0} /(1-b)$ may be big even when $k r_{0} \ll 1$. Thus we will need to consider the limits $b \lesssim 1$ and $b \rightarrow 1$ separately. We have already encountered this previously, in the example with shock waves. With this in mind, we see that the consistent approximation of the Green's functions (111) and (112) is

$$
\begin{aligned}
\mathcal{G}_{\mathrm{TT}} & =\frac{1}{M_{6}^{4}} \frac{1}{\frac{K_{1}\left(k \frac{r_{0}}{1-b}\right)}{K_{0}\left(k \frac{0}{1-b}\right)} k+\frac{M_{5}^{3}}{M_{6}^{4}} k^{2}}, \\
\mathcal{G}_{\mathrm{X}} & =\frac{1}{M_{6}^{4}} \frac{1}{\frac{K_{1}\left(k \frac{r_{0}}{1-b}\right)}{K_{0}\left(k \frac{r_{0}}{1-b}\right)} k+\frac{M_{5}^{3}}{M_{6}^{4}}\left[k^{2}+\frac{3 b M_{6}^{4}}{4 M_{5}^{3} r_{0}}-\beta\left(k^{2}\right)\right]},
\end{aligned}
$$

The TT-tensor sector is particularly simple to understand from (112). As with shock waves, we see that the behavior of the theory is governed by the ratio $k_{c}=\frac{M_{6}^{4} K_{1}\left(k \frac{r_{0}}{1-b}\right)}{M_{5}^{3} K_{0}\left(k \frac{r_{0}}{1-b}\right)}$, which controls the denominator of $\mathcal{G}_{\text {Tт }}$ at low momenta. For $k<k_{c}$, or distances $\ell>1 / k_{c}$, the dominant contributions always come from $\propto \frac{K_{1}\left(k \frac{r_{0}}{1-b}\right)}{K_{0}\left(k \frac{r_{0}}{1-b}\right)}$. In this limit the long range fields manifestly reveal the extra dimensions, since the scaling of the potentials will not be $4 D$. In the generic sub-critical cases, $b \lesssim 1$ and for momenta $k \ll 1 / r_{0}$ we can replace the Bessel 
functions $K_{\nu}$ by their small argument expansion along the brane, which yields the crossover scale identical to Eq. $(52), r_{c}^{2}(k)=\frac{M_{4}^{2}}{2 \pi(1-b) M_{6}^{4}} \ln \left[\frac{2(1-b)}{k r_{0}}\right]$. Beyond this distance, the theory behaves as a $6 D$ gravity on a cone.

In the near-critical limit, however, $b \rightarrow 1$, at intermediate momenta we should instead approximate $K_{\nu}$ by their large argument expansion, as in the shock wave analysis of the nearcritical models building up to Eq. (56). In this case the crossover scale is $r_{c}=\frac{M_{5}^{3}}{M_{6}^{4}}$, where gravity first changes into a $5 D$ theory, because for near-critical tensions one bulk dimension is efficiently compactified on a circle, as discussed recently in [59], and in our shock wave analysis. Eventually, the circle opens up and the theory again turns into a $6 D$ gravity on a cone. As a result we see that the crossover scale, beyond which the theory will not look $4 D$, is exactly the same as in Eq. (59), which was already revealed by the shock waves. We again confirm that the see-saw mechanism of [29] is realized only in the near-critical limit, but the crucial dynamics which manufactures the see-saw scale is the compactification of one bulk dimension induced by a near-critical brane [59].

What remains is to check precisely what kind of $4 D$ theory we have below the crossover scale $r_{c}$, in the regime of distances $r_{0} \ll \ell \ll r_{c}$. For TT-tensors, by previous discussion we can neglect the terms in the denominator $\sim \frac{K_{1}\left(k \frac{r_{0}}{1-b}\right)}{K_{0}\left(k \frac{r_{0}}{1-b}\right)}$. The Green's function reduces to $\mathcal{G}_{\text {TT }} \rightarrow \frac{1}{M_{5}^{3} k^{2}}$. The non-relativistic matter sources on the wrapped brane are described by stress energy of Eq. (35), which Fourier-transforms to $\tau_{b}^{a}=-\mu \operatorname{diag}(1,0,0,0,1)$. So the leading order solution for the TT-tensor field is

$$
\gamma_{\nu}^{\mu}{ }_{\nu}(k, \rho)=\frac{2 \mu}{3 M_{5}^{3} k^{2}} \begin{cases}2, & \mu=\nu=0, \\ -\left(\delta^{j}{ }_{k}-\frac{k^{j} k_{k}}{k^{2}}\right), & \mu=j, \nu=k .\end{cases}
$$

Next we need to evaluate the Fourier integrals $\gamma^{\mu}{ }_{\nu}(\vec{x})=\int \frac{d^{3} \vec{k}}{(2 \pi)^{3}} \gamma^{\mu}{ }_{\nu}(\vec{k}) e^{i \vec{k} \cdot \vec{x}}$. Since a Fourier integral picks up the dominant contribution from the momenta $k \sim 1 /|\vec{x}|$, in the $4 D$ regime, when $|\vec{x}|<r_{c}$, we can approximate $\gamma^{\mu}{ }_{\nu}(k)$ by (116). The remaining integral is easy for $\gamma_{0}^{0}$, and yields ${ }^{3} \gamma_{0}^{0}=\frac{\mu}{3 \pi M_{5}^{3}|\vec{x}|}$ in this regime. Recalling that $\mu$ is the mass per unit length of string (see Eq. (31)), so that $\mu=\frac{\mathcal{M}}{2 \pi r_{0}}$, and using as before Gauss law to trade the $5 D$ Planck scale $M_{5}$ for the $4 D$ one, $M_{5}^{3}=\frac{M_{4}^{2}}{2 \pi r_{0}}$, we finally obtain

$$
\gamma_{0}^{0}(\vec{x})=\frac{\mathcal{M}}{3 \pi M_{4}^{2}|\vec{x}|} .
$$

To find $\gamma_{k}^{j}$, we don't need to do the Fourier integrals directly. Instead, using Lorentz invariance, we see from (116) that $\gamma^{j}{ }_{k}=\frac{\mathcal{C}_{1}}{|\vec{x}|}\left(\delta^{j}{ }_{k}+\mathcal{C}_{2} \frac{x^{j} x_{k}}{\vec{x}^{2}}\right)$. Then since $\gamma^{\mu}{ }_{\nu}$ is a TT-tensor, and $\gamma_{0}^{j}=0$, using transversality $\partial_{j} \gamma^{j}{ }_{k}=0$, we get $\mathcal{C}_{2}=1$. Further vanishing trace $\gamma^{\mu}{ }_{\mu}=0$ implies $\gamma_{k}^{k}=-\gamma_{0}^{0}$, which by comparing with Eq. (117) sets $\mathcal{C}_{1}=-\frac{\mathcal{M}}{12 \pi M_{4}^{2}}$. Thus we find

$$
\gamma_{k}^{j}(\vec{x})=-\frac{\mathcal{M}}{12 \pi M_{4}^{2}|\vec{x}|}\left(\delta^{j}{ }_{k}+\frac{x^{j} x_{k}}{\vec{x}^{2}}\right) .
$$

\footnotetext{
${ }^{3} \mathrm{U} \operatorname{sing} \int \frac{d^{3} \vec{k}}{(2 \pi)^{3}} \frac{e^{i \vec{k} \cdot \vec{x}}}{\vec{k}^{2}}=\frac{1}{4 \pi|\vec{x}|}$.
} 
It is convenient to rewrite the solutions by introducing the effective $4 D$ Newton's constant $G_{N \text { eff }}=\frac{1}{8 \pi M_{4}^{2}}$. Then, in the leading order for $|\vec{x}|<r_{c}$,

$$
\begin{aligned}
\gamma_{0}^{0}(\vec{x}) & =\frac{8}{3} G_{N \text { eff }} \frac{\mathcal{M}}{|\vec{x}|} \\
\gamma_{k}^{j}(\vec{x}) & =-\frac{2}{3} G_{N \text { eff }} \frac{\mathcal{M}}{|\vec{x}|}\left(\delta^{j}{ }_{k}+\frac{x^{j} x_{k}}{\vec{x}^{2}}\right) .
\end{aligned}
$$

If we were to ignore the scalar field $X$ for the moment, the Newtonian potential $V_{N}=-h^{0}{ }_{0} / 2$ would be $V_{N}=-\frac{4}{3} G_{N \text { eff }} \frac{\mathcal{M}}{|\vec{x}|}$, which is a factor of $4 / 3$ larger than the usual formula of General Relativity. This is a manifestation of the Iwasaki-van Dam-Veltman-Zakharov discontinuity [17], which signals the presence of the helicity-0 modes in the graviton multiplet. The factor $4 / 3$ enhancement is precisely what one expects based on other examples [15], and in this case shows that the extra helicity- 0 modes in the spin- 2 multiplet mediate attractive force, just as the helicity-2 modes. Thus, they are not ghosts.

The scalar Green's function $\mathcal{G}_{\mathrm{X}}$ is considerably more involved because of the function $\beta\left(k^{2}\right)$ in the denominator. It behaves differently for generic sub-critical and for near-critical branes, and so we need to deal with it with some care. First off, we note that the terms $k^{2}+\frac{3 b M_{6}^{4}}{4 M_{5}^{3} r_{0}}-\beta\left(k^{2}\right)$ factorize as

$$
k^{2}+\frac{3 b M_{6}^{4}}{4 M_{5}^{3} r_{0}}-\beta\left(k^{2}\right)=\frac{r_{0}^{2} k^{2}}{1-b+r_{0}^{2} k^{2}}\left[\left(1-\frac{3(1-b) M_{5}^{3}}{4 b M_{6}^{4} r_{0}}\right) k^{2}+\left(\frac{3 b M_{6}^{4}}{4 M_{5}^{3} r_{0}}-\frac{1-b}{2 r_{0}^{2}}\right)\right] .
$$

After substituting this in Eq. (115) we find that $\mathcal{G}_{\mathrm{x}}$ reduces to

$$
\mathcal{G}_{\mathrm{X}}=\frac{1}{M_{6}^{4}} \frac{1}{\frac{K_{1}\left(k \frac{r_{0}}{1-b}\right)}{K_{0}\left(k \frac{r_{0}}{1-b}\right)} k+\frac{M_{5}^{3}}{M_{6}^{4}} \frac{r_{0}^{2} k^{2}}{1-b+r_{0}^{2} k^{2}}\left[\left(1-\frac{3(1-b) M_{5}^{3}}{4 b M_{6}^{4} r_{0}}\right) k^{2}+\left(\frac{3 b M_{6}^{4}}{4 M_{5}^{3} r_{0}}-\frac{1-b}{2 r_{0}^{2}}\right)\right]} .
$$

Now, we will generically require $r_{0} \ll M_{5}^{3} / M_{6}^{4}$ in order to have the crossover scale for gravity be much larger than the radius of the compact dimension, $r_{c} \gg r_{0}$. Otherwise, we can never get the theory to behave as a $4 D$ gravity.

For generic sub-critical branes, $b \lesssim 1$, at scales $k \ll 1 / r_{0}$ the scalar Green's function (121) is approximated by

$$
\mathcal{G}_{\mathrm{x}}=\frac{1}{M_{6}^{4}} \frac{r_{0} \ln \left[\frac{2(1-b)}{k r_{0}}\right]}{1-b-\frac{3 M_{5}^{6} r_{0}^{2}}{4 b M_{6}^{8}} k^{2}\left(k^{2}+\frac{2 b M_{6}^{4}}{3 M_{5}^{3} r_{0}}\right) \ln \left[\frac{2(1-b)}{k r_{0}}\right]},
$$

where we have approximated $\frac{K_{1}\left(k \frac{r_{0}}{1-b}\right)}{K_{0}\left(k \frac{r_{0}}{1-b}\right)}$ with the small argument expansion. Hence at very large distances, or low momenta, the scalar Green's function depends on the momentum only logarithmically, $\mathcal{G}_{\mathrm{X}} \simeq \frac{r_{0} \ln \left[\frac{2(1-b)}{k r_{0}}\right]}{(1-b) M_{6}^{4}}$, implying that the configuration space solution for the scalar field $X$ depends on the distance as $X \sim \mathcal{M} /|\vec{x}|^{3}$ - i.e. as a field in $6 D$. However, because the momentum-dependent terms in the denominator of $\mathcal{G}_{\mathrm{X}}$ are negative, as we move 
inwards towards the source, the scalar force grows, and the scalar eventually becomes strongly coupled as $k$ approaches the pole of (122). This happens when $k \sim k_{*}$, where

$$
k_{*}^{2} \simeq \frac{2(1-b) M_{6}^{4}}{M_{5}^{3} r_{0} \ln \left[\frac{2(1-b)}{k r_{0}}\right]} .
$$

But this is essentially the same as the crossover momentum in the TT-tensor sector, as we can see by comparing $k_{*}$ with $k_{c}$ given in Eq. (50), after recalling that $M_{4}^{2} \simeq M_{5}^{3} r_{0}$. At first sight, it looks a little surprising that for resolved codimension-2 branes the strongly coupled scalars appear even around the vacuum, in contrast to the codimension-1 brane induced gravity models, as discussed in [19, 20, 21, 22, 23]. However the $4 D$ vacua aren't really vacuum solutions from the point of view of the full brane worldvolume theory because they contain the flux of the axion $\Sigma$ and hence source the brane bending term $\xi$ even in the absence of localized matter sources, as seen in Eq. (99). This in turn triggers the onset of strong coupling.

Thus we see that for sub-critical branes with $b \lesssim 1$, inside the regime of length scales $\ell<r_{*}$ where

$$
r_{*}^{2} \simeq \frac{M_{4}^{2}}{2(1-b) M_{6}^{4}} \ln \left[\frac{2(1-b)}{k r_{0}}\right],
$$

the TT-tensor is $4 D$, but with the wrong tensor structure, as we have discussed following Eq. (119), however the scalar sector is in fact strongly coupled. This makes the perturbation theory around the vacuum on sub-critical branes completely unreliable inside the regime where gravity might be $4 D$. The negative signs of the momentum-dependent terms in the denominator of (122) might naively suggest presence of ghosts in this regime, but at this level of the approximation we cannot conclude that decisively. One exception to the pathological behavior of the theory is the limit of relativistic sources, for which $\tau^{\mu}{ }_{\mu}=\tau_{\phi}^{\phi}=0$, so that the scalars are never sourced and the tensor sector reduces to the $4 D$ Aichelburg-Sexl solution below the crossover scale. One needs some means for exploring the scalar sector beyond linearized theory, perhaps along the lines of [68], or by seeking exact solutions, before passing on the final verdict on the sub-critical brane models. At the level of linearized perturbation theory the gravitational effects below the crossover scale are not calculable.

In the near-critical limit the scalar sector behaves very differently. At distances $\ell \gg r_{0}$, where $k r_{0} \simeq \frac{r_{0}}{\ell} \ll 1$ the argument of the Bessel functions $K_{\nu}$ will still be very large when $b \rightarrow 1$. We have already noted this in the discussion of shock waves. Hence for distances below the crossover scale we can use the large argument expansion for $K_{\nu}$ 's, which yields $K_{1} \rightarrow K_{0}$, as long as $r_{0} k \gg 1-b$. Moreover, we can immediately neglect the negative terms in the denominator of $\mathcal{G}_{\mathrm{X}}$ in Eq. (121) because $\frac{(1-b) M_{5}^{3}}{M_{6}^{4} r_{0}}=(1-b) \frac{r_{c}}{r_{0}}<1$. The scalar Green's function is therefore

$$
\mathcal{G}_{\mathrm{X}}=\frac{1}{M_{6}^{4}} \frac{1}{k+\frac{M_{5}^{3}}{M_{6}^{4}} \frac{r_{0}^{2} k^{2}}{1-b+r_{0}^{2} k^{2}}\left(k^{2}+\frac{3}{4 r_{c} r_{0}}\right)} .
$$

where $r_{c}=M_{3}^{5} / M_{6}^{4}$ is the TT-tensor crossover scale, as per Eq. (56). Now, it is convenient to define the scale $r_{\text {vac }}$ by

$$
1-b=\frac{r_{0}^{2}}{r_{\mathrm{vac}}^{2}}
$$


In the limit $b \rightarrow 1$ we have $r_{\text {vac }}=\sqrt{1-b} \frac{r_{0}}{1-b} \ll \frac{r_{0}}{1-b}$, and so the scale $r_{\text {vac }}$ is always smaller than the size of the conical throat surrounding the brane. This is why the scale $r_{\text {vac }}$ may compete with $r_{c}$ for the control over the scalar sector. Both $r_{\mathrm{vac}}$ and $r_{c}$ are smaller than the size of the throat, and the details of sub-crossover dynamics depend on their ratio. First, note that for momenta $k$ such that $r_{\text {vac }} k>1$, we can expand $\frac{r_{0}^{2} k^{2}}{1-b+r_{0}^{2} k^{2}} \simeq 1+\mathcal{O}\left(\frac{1-b}{r_{0}^{2} k^{2}}\right)$ and keep only the first term. The scalar Green's function in this limit at momenta $r_{0} k<1-b$ reduces to

$$
\mathcal{G}_{\mathrm{X}}=\frac{1}{M_{5}^{3}} \frac{1}{\frac{k}{r_{c}}+k^{2}+\frac{3}{4 r_{c} r_{0}}} .
$$

Thus for momenta $k>\max \left(1 / r_{c}, 1 / r_{\text {vac }}\right)$, the theory looks $4 D$, but the scalar has a mass term, $m_{\mathrm{x}}^{2}=\frac{3}{4 r_{c} r_{0}}$. Therefore its long range effects have a Yukawa suppression $\propto \exp \left(-m_{\mathrm{x}} r\right)$. Hence in the range of scales $\frac{1}{m_{\mathrm{x}}}<\ell<\min \left(r_{c}, r_{\mathrm{vac}}\right)$ the scalar field exchange will lead to negligible, exponentially suppressed effects compared to the TT-gravitons. This mass term is completely analogous to a brane-localized mass term of a bulk scalar. In such models the scalar is repelled from the brane, since it prefers to reside in the region of space where its inertia is minimized. This resembles Meissner effect in superconductivity, where the mass term pushes the magnetic field outside of the superconducting medium. In our case however, the scalar is also pulled back to the brane by the brane-localized kinetic terms, and the result is the Yukawa suppression alone, which is manifest in (127) in the same coupling $1 / M_{5}^{3}$ as for the TT-tensors below the crossover scale, e.g. in Eq. (116). Similar phenomena were also studied recently for gauge fields in gaugephobic models [69].

Although $X$ may be Yukawa-suppressed, so its direct long range forces will be small compared to those arising from the TT-tensor sector, the brane bending term $\xi$ may yet yield a long range tail, as is clear from Eq. (99). We see that $\xi$ is proportional to $k^{2} X$, and this will in general lead to long range effects that may dominate the Yukawa-suppressed effect from direct $X$-exchange. It turns out that the $\xi$-induced effects will be parametrically smaller than the dominant TT-tensor fields, as we will show below.

At distances $\ell>r_{c}$, the linear term in $k$ in the denominator of (127) dominates the momentum transfer in scalar exchange, modifying the theory to a $5 D$ one, and eventually, after resuming the corrections which we neglected in setting $K_{1} / K_{0} \simeq 1$, one would see how the theory changes to $6 D$. When $r_{\mathrm{vac}}>r_{c}$ the story of the scalar dynamics ends here.

However, when $r_{\text {vac }}<r_{c}$, in the regime of scales $r_{\text {vac }}<\ell<r_{c}$ below the crossover scale, we can approximate the fraction in the denominator of (125) by $\frac{r_{0}^{2} k^{2}}{1-b+r_{0}^{2} k^{2}} \simeq \frac{r_{0}^{2} k^{2}}{1-b}$. Thus, $\mathcal{G}_{\mathrm{X}}{ }^{-1} \sim k+\frac{M_{5}^{3} r_{0}^{2}}{(1-b) M_{6}^{4}} k^{2}\left(k^{2}+\frac{3}{4 r_{c} r_{0}}\right)$. Then, since the mass term $m_{\mathrm{x}}^{2}$ is larger than the momenta in this regime, because $k^{2}<1 / r_{\text {vac }}^{2}$ so that $\frac{k^{2}}{m_{\mathrm{x}}^{2}}<\frac{4 r_{c} r_{0}}{3 r_{\text {vac }}^{2}}=(1-b) \frac{4 r_{c}}{3 r_{0}}<1$, the Green's function is approximately

$$
\mathcal{G}_{\mathrm{X}}=\frac{1}{M_{6}^{4}} \frac{1}{k+\frac{3 r_{\mathrm{vac}}^{2}}{4 r_{0}} k^{2}} .
$$

What happened here is that due to the momentum dependence of the effective coupling, the scalar field mass term got overcompensated and the field became essentially massless all the 
way out to a new scalar crossover scale, given by (using Eq. (126))

$$
\mathcal{R}_{c}=\frac{3}{4} \frac{r_{\mathrm{vac}}^{2}}{r_{0}}=\frac{3}{4} \frac{r_{0}}{1-b}
$$

which is essentially equal to the length of the throat inside which bulk gravity looks $5 D$. Below $\mathcal{R}_{c}$, the scalar propagator reduces to $\mathcal{G}_{\mathrm{X}} \rightarrow \frac{4 r_{0}}{3 M_{6}^{4} r_{\mathrm{vac}}^{2} k^{2}}<\frac{1}{M_{5}^{3} k^{2}}$, because $r_{c}<\frac{r_{0}}{1-b}$ in the near-critical limit. Thus, when $r_{\text {vac }}>r_{c}$, the scalar remains massless all the way out to $\mathcal{R}_{c}$, but below the crossover scale it couples more weakly than the TT-tensor.

We can now put together the various contributions to the fields of a static massive ring on the brane at distances below the crossover scale. The scalar Green's function for near-critical branes below the crossover scale is well approximated by

$$
\mathcal{G}_{\mathrm{X}}= \begin{cases}\frac{1}{M_{5}^{3}} \frac{1}{k^{2}+m_{\mathrm{x}}^{2}}, & r_{c}<r_{\mathrm{vac}}, \frac{1}{r_{c}}<k \\ \frac{4(1-b)}{3 M_{6}^{4} r_{0}} \frac{1}{k^{2}}, & r_{\mathrm{vac}}<r_{c}, \frac{1}{r_{c}}<k<\frac{1}{r_{\mathrm{vac}}} .\end{cases}
$$

The scalar field $X$ sourced by a ring of mass with Fourier-transformed stress energy tensor $\tau_{b}^{a}=-\mu \operatorname{diag}(1,0,0,0,1)$ is then, using the solution (104) for this range of scales, and again replacing $\mu=\frac{\mathcal{M}}{2 \pi r_{0}}$ and $M_{5}^{3}=\frac{M_{4}^{2}}{2 \pi r_{0}}$ as in the earlier discussion of TT-tensors,

$$
X= \begin{cases}-\frac{\mathcal{M}}{3 M_{4}^{2}} \frac{1}{k^{2}+m_{\mathrm{x}}^{2}}, & r_{c}<r_{\mathrm{vac}}, \frac{1}{r_{c}}<k, \\ \frac{2 \mathcal{M}}{9 M_{4}^{2}} \frac{(1-b) r_{c}}{r_{0}} \frac{1}{k^{2}}, & r_{\mathrm{vac}}<r_{c}, \frac{1}{r_{c}}<k<\frac{1}{r_{\mathrm{vac}}},\end{cases}
$$

while the brane bending, normalized to $r_{0}$, is, from (99),

$$
\frac{\xi}{r_{0}}= \begin{cases}-\frac{r_{c} r_{0}}{r_{\mathrm{vac}}^{2}} \frac{\mathcal{M}}{6 M_{4}^{2}}\left(\frac{10}{k^{2}}-\frac{1}{k^{2}+m_{\mathrm{x}}^{2}}\right), & r_{c}<r_{\mathrm{vac}}, \frac{1}{r_{c}}<k, \\ \frac{(1-b) r_{c}}{r_{0}} \frac{\mathcal{M}}{3 M_{4}^{2}} \frac{1}{k^{2}}+\text { constant }, & r_{\mathrm{vac}}<r_{c}, \frac{1}{r_{c}}<k<\frac{1}{r_{\mathrm{vac}}} .\end{cases}
$$

The constant in the brane bending transforms to a gauge-dependent contact term $\propto \delta^{(3)}(\vec{x})$, which is ultralocal and hence we can neglect it altogether. The configuration space solutions ${ }^{4}$ for the scalars $X$ and $\xi$ are therefore

$$
X= \begin{cases}-\frac{\mathcal{M}}{12 \pi M_{4}^{2}|\vec{x}|} e^{-m_{\mathrm{x}}|\vec{x}|}, & |\vec{x}|<r_{c}<r_{\mathrm{vac}}, \\ \frac{(1-b) r_{c}}{r_{0}} \frac{\mathcal{M}}{18 \pi M_{4}^{2}|\vec{x}|}, & r_{\mathrm{vac}}<|\vec{x}|<r_{c}\end{cases}
$$

and

$$
\frac{\xi}{r_{0}}= \begin{cases}-\frac{r_{c} r_{0}}{r_{\text {vac }}^{2}} \frac{\mathcal{M}}{24 \pi M_{4}^{2}|\vec{x}|}\left(10-e^{-m_{\mathrm{x}}|\vec{x}|}\right), & |\vec{x}|<r_{c}<r_{\mathrm{vac}} \\ \frac{(1-b) r_{c}}{r_{0}} \frac{\mathcal{M}}{12 \pi M_{4}^{2}|\vec{x}|}, & r_{\mathrm{vac}}<|\vec{x}|<r_{c} .\end{cases}
$$

Now, in the case when $r_{c}<r_{\text {vac }}$, the scalar field $X$ will have a very short range due to its mass. Indeed, since $m_{\mathrm{vec}}^{-2} \sim r_{0} r_{c}$ is the geometric mean between the brane radius and the crossover scale, $m_{\mathrm{vec}}^{-1}$ will be quite small. In fact, if the brane size is set by the

${ }^{4}$ Which we find using $\int \frac{d^{3} \vec{k}}{(2 \pi)^{3}} \frac{e^{i \vec{k} \cdot \vec{x}}}{k^{2}+m_{\mathrm{x}}^{2}}=\frac{1}{4 \pi|\vec{x}|} e^{-m_{\mathrm{x}}|\vec{x}|}$. 
$4 D$ Planck length and the crossover scale by the current horizon size, $r_{0} \sim 1 / M_{4}$, and $r_{c} \sim 1 / H_{0}$ respectively, $m_{\mathrm{x}}^{-1}$ will be near the table top bounds of about $0.1 \mathrm{~mm}$. Thus $X$ is de facto decoupled at the scales where we normally probe $4 D$ gravity. On the other hand, the brane bending can still simulate a long range effect which scales as $1 /|\vec{x}|$ due to the momentum dependence of the couplings. However, the strength of its contributions is a factor $\frac{r_{c} r_{0}}{r_{\text {vac }}^{2}}=\frac{r_{c}}{r_{\text {vac }}} \frac{r_{0}}{r_{\text {vac }}} \ll 1$ down compared to the TT-tensor contributions in Eq. (119). In the case when $r_{\mathrm{vac}}<r_{c}$, the scalars remain essentially massless and source long range fields. However, their strength is still suppressed, this time by $\frac{(1-b) r_{c}}{r_{0}} \ll 1$, by definition of the near-critical limit. While the scalar effects become more important as this factor becomes smaller, so do the strong coupling effects which we cannot neglect away from the near-critical limit. Indeed, as $\frac{(1-b) r_{c}}{r_{0}} \rightarrow 1$, where the brane bending effects could compete with the long range fields of TT tensors, the strong coupling effects become very important, and we lose calculability in linearized perturbation theory below the crossover scale.

Hence in either case the long range effects of the scalars in linearized perturbation theory around the near-critical vacuum cannot compete with the TT-tensors, as long as the perturbative treatment is valid. We can therefore ignore the scalars in the formula (108). The long range fields are determined by $\gamma^{\mu}{ }_{\nu}$ alone, which are given in Eq. (119). Clearly, these solutions do not look like $4 D$ General Relativity. Instead, in the leading order they really mimic a scalar tensor theory, due to the presence of the helicity-0 contributions. To see this, we can compare the solutions (119) with the spherically symmetric linearized solution in the PPN approximation [64]. For the purpose of this comparison, we define $\hat{G}_{N}=\frac{4}{3} G_{N \text { eff }}$, so that

$$
\begin{aligned}
\gamma_{0}^{0} & =2 \hat{G}_{N} \frac{\mathcal{M}}{|\vec{x}|} \\
\gamma_{k}^{j} & =-\frac{1}{2} \hat{G}_{N} \frac{\mathcal{M}}{|\vec{x}|} \delta^{j}{ }_{k}-\frac{1}{2} \hat{G}_{N} \frac{\mathcal{M} x^{j} x_{k}}{|\vec{x}|^{3}} .
\end{aligned}
$$

Comparing to [64] (taking into account overall sign difference reflecting our conventions) we find that the solution (135) mimics a Brans-Dicke theory with $\gamma=\frac{1}{2}$, or therefore, with the Brans-Dicke $\omega$ parameter equal to zero, where the parameter $\omega$ is defined in the usual way in the Brans-Dicke action as $S_{B D}=\int d^{4} x \sqrt{g}\left(\Phi R-\omega(\nabla \Phi)^{2} / \Phi\right)$. This theory is in conflict with observational data as it stands, and hence is not a realistic description of our Universe.

However, it is possible that non-linear effects, which we have neglected throughout this work, could play a role here. Indeed, even in conventional General Relativity, nonlinearities start to show up at distances comparable with the gravitational radii of the sources. In brane induced gravity, and other frameworks that strive to modify gravity in the IR, the nonlinear corrections will become important even sooner than the nonlinearities in General Relativity $[18,19,20,21,22,23]$. Our results are found in linearized perturbation theory around a near-critical vacuum, and so it is conceivable (although not certain) that different strong coupling effects at higher orders in perturbation theory might improve the behavior of long range fields of masses far from the source but well below the crossover scale. Alternatively, it is possible that additional tweaks of the bulk theory, for example by curving the bulk locally, could change how the helicity-0 mode couples to brane matter. Such methods are widely used in the construction of string landscapes [3], and might also be useful here. 
We stress again that regardless of the ratio of $r_{\mathrm{vac}} / r_{c}$ the scalar couplings remain consistently weak for static sources on near-critical branes with $b \rightarrow 1$. The static solutions are governed by Euclidean momenta, and so they are always finite in linearized theory around near-critical vacua, in contrast to the fields of masses which perturb generic sub-critical vacua. Thus linearized perturbation theory remains under control on near-critical vacua. This as we already mentioned does not guarantee that the linearized solutions will remain dominant close to the masses that source the fields. Further study of these issues, to ascertain how likely it is that nonlinear phenomena may yield any additional screening of helicity-0 modes, hence seems warranted. The near-critical vacua at the very least provide us with a controllable new arena where such phenomena could be studied.

The near-critical propagating solutions, on the other hand, also remain under control, albeit their dynamics is more subtle. The issue is that there may be new poles at Lorentzian momenta $k^{2}=-k_{\text {vac }}^{2}=-\frac{1-b}{r_{0}^{2}}$, in the solutions for $\xi$ and $X$, given respectively by Eqs. (99), (104) and (112), and their approximations below the crossover scales. Clearly, these poles never play any role for static configurations, which are controlled by Euclidean momenta for which the fields remain finite. However one may worry if new infinities could plague the linearized theory once a weak time dependence is allowed. Now, on a generic sub-critical brane the poles at $k^{2}=-k_{\text {vac }}^{2}$ reside practically on the $4 D$ cutoff $\sim 1 / r_{0}$, deep in the strongly coupled regime of the sub-critical linearized theory and hence can be completely ignored at large distances as a UV mirage. On the other hand, in the near-critical limit $b \rightarrow 1$ the scale $k_{\text {vac }}$ becomes very low, and may be much lower than the crossover scale $1 / r_{c}$. However, as we will now explain, in this case these poles are harmless because they merely point to a breakdown of a gauge fixing of the linearized theory, which is fixed by an interchange of scalar modes. The easiest way to see this is to reconsider the formula for the $\xi$ field, Eq. (99), and the boundary condition for $\Delta X^{\prime}$, Eq. (100), when Lorentzian momentum is $k^{2}=-k_{\text {vac }}^{2}=-\frac{1-b}{r_{0}^{2}}$. Then, Eq. (99) indicates that $\xi$ may diverge; however since the brane bending must be bounded, this equation must be reinterpreted by multiplying it by $1-b+r_{0}^{2} k^{2}$ before setting $k^{2}=-k_{\text {vac }}^{2}$, and then demanding that $\xi$ is finite, which means that the RHS must vanish identically. This fixes $X$ on the brane to

$$
\left.X\right|_{k^{2}=-k_{\text {vac }}^{2}}=\left.\frac{2 r_{0}^{2}}{3 M_{5}^{3}\left(1-b-\frac{b M_{6}^{4} r_{0}}{M_{5}^{3}}\right)} \tau_{\phi}^{\phi}\right|_{k^{2}=-k_{\text {vac }}^{2}},
$$

while now $\xi$ is not determined by this boundary condition. Since $X$ is now fixed by the matter source, it cannot be freely chosen to satisfy the boundary condition for $\Delta X^{\prime}$ in (100). This is what fixes $\xi$ : indeed, using (99) and (101) we can rewrite (100) as

$$
\left.\Delta X^{\prime}\right|_{k^{2}=-k_{\mathrm{vac}}^{2}}=\left.\frac{M_{5}^{3}}{M_{6}^{4}}\left[\left(1-b-\frac{b M_{6}^{4} r_{0}}{M_{5}^{3}}\right) \frac{\xi}{2 r_{0}^{3}}-\frac{1-b}{1-b-\frac{b M_{6}^{4} r_{0}}{M_{5}^{3}}} \frac{\tau_{\phi}^{\phi}}{6 M_{5}^{3}}-\frac{\tau_{\alpha}^{\alpha}}{6 M_{5}^{3}}\right]\right|_{k^{2}=-k_{\mathrm{vac}}^{2}},
$$

so that for given sources and for $X$ fixed by (136) when $k^{2}=-\frac{1-b}{r_{0}^{2}}$ we can choose $\xi$ to satisfy (137). Therefore the Lorentzian poles at $k^{2}=-k_{\text {vac }}^{2}$ are spurionic, and can be ignored in the linearized perturbation theory on near-critical branes. The linearized theory around the vacuum on such generic near-critical backgrounds remains under control, without dramatic 
instabilities, and mimicking Brans-Dicke theory with $\omega=0$, although we stress again that one has to reevaluate it against the higher order corrections closer to the source, because of the issues related to the Vainshtein scale and strong coupling at higher orders.

\section{Conclusions}

Our main result is the observation that properly regulated brane induced gravity theories, which yield calculable long distance gravitational fields, really behave as a semiclassical landscape of vacua. Treating gravity classically, these models can impersonate $4 D$ worlds between the UV cutoff that resolves the core of the gravity-localizing defect and the crossover scale, if their Scherk-Schwarz sector is carefully tuned to yield $4 D$ Minkowski vacua. While we have explicitly worked with codimension- 2 defects, we feel that similar conclusions should extend to any setups with codimension $\geq 2$. In the case of codimension- 2 , we see that the brane vacua can remain completely flat, while the bulk readjusts to absorb the tension as deficit angle. Separating them there will be mismatched configurations, where the tensional pressure in the compact direction may not be precisely cancelled, which are either nonstationary, or never approximate $4 D$ behavior. Static supercritical branes must be singular, just like supercritical local strings in $4 D$ [54]. However in this case there should exist nonsingular inflating solutions, that should really be the correct vacua for supercritical branes.

We also find that the cosmological constant problem changes its guise rather dramatically in codimension-2 setups. Although the theory has many $4 D$ flat vacua with Minkowski metric, the effective $4 D$ Planck scale, and for sub-critical tensions, the crossover scale out to which gravity looks $4 D$, are very sensitive to the brane tension. These parameters depend on the tension directly, as does the sub-critical crossover scale (see Eq. (59)) or through the compactification radius of the wrapped 4-brane, as does the effective $4 D$ Planck scale. So if the theory is to mimic a weak $4 D$ gravity over a large spatial region, which looks flat and static, for a given brane and bulk Planck scales $M_{5}$ and $M_{6}$ and a fixed brane radius $r_{0}$ one must tune the brane tension precisely. Changes of brane tension will generically lead to proportional changes of the crossover scale and $4 D$ gravitational coupling, requiring appropriate retunings. However in the near-critical limit, where the bulk compactifies to a cone so that $4 D$ gravity first changes to a $5 D$ one, the crossover scale saturates at a value completely independent of the tension, $r_{c}=M_{5}^{3} / M_{6}^{4}$. To get this to be of the order of the current horizon size, $H_{0}^{-1} \sim 10^{28} \mathrm{~cm}$, and ensure that the rings of matter on the brane look pointlike at energies below a TeV, one needs $M_{6} \gtrsim \mathrm{TeV}$, and therefore $M_{5} \lesssim 10^{19} \mathrm{GeV}$. Further to reproduce the $4 D$ Newton's constant below the crossover scale, one needs to pick

$r_{0} \gtrsim 10^{-19} \mathrm{GeV}^{-1}$. These numbers are rather curious. Clearly, one must tune the theory to make sure that the brane is very thin, with many hidden sector fields, to get such hierarchies. We don't have much to add to the discussion of how to do it, but merely note that this is in line with the current philosophy of the brane induced gravity models [15]. While some work along the lines of embedding the theory into string theory, that would provide the framework for its full UV completion, has been pursued [60, 61, 62], the existing constructions are really semiclassical models where gravity is treated classically. Adopting this possibility, one may at least explore the low energy consequences of such models. 
Once we consider gravity of localized static masses on the brane, we find that for subcritical tensions there is a strong coupling scale in the vacuum itself, which is essentially the same as the crossover scale. Thus in the regime where gravity may appear $4 D$, linearized perturbation theory does not apply because the scalar modes are strongly coupled. However in the near-critical limit the linearized perturbation theory around the vacuum is under control. We find that it does not contain instabilities, and generates long range fields which are, to the leading order, the same as in the Brans-Dicke theory with $\omega=0$. This would be in conflict with tests of gravity in Solar System and beyond. However, throughout this work we have taken the bulk to be locally flat. Adding bulk fields and curvature will increase the diversity of possible solutions and might yield additional effects screening the helicity-0 mode in the spin-2 sector, perhaps similarly to what happens in string landscape constructions [3]. Moreover, the nonlinear corrections might play some role too as advocated in $[18,19]$.

This discussion shows that many questions remain open. Clearly, the most interesting phenomenological issues concern the stabilization or decoupling of the helicity-0 modes. It is interesting to explore the theory for dangerous instabilities beyond the linear order. It also remains to see if the brane induced gravity models can be consistently derived from microscopic models that admit plausible UV completions, generating $M_{4} \gg M_{6}$. In other words, do such landscapes even exist beyond a classical action, that one can write for them? Finally, it would be interesting to see if the new guise of the cosmological constant problem, and in particular the serendipitous insensitivity of the crossover scale from brane tension in the near-critical limit, might yield some new avenues for going around the venerated Weinberg no-go theorem [1], that is still the main obstruction to having a mechanism for protecting a small $4 D$ vacuum curvature from quantum radiative corrections. These issues are outside of the scope of the present work, and we can only hope that our sketch of such a diverse new landscape with a different manifestation of the vacuum energy problem may motivate the search for their resolutions.

\section{Acknowledgements}

We would like to thank Alberto Iglesias, Robert Myers, Minjoon Park, Oriol Pujolas and Lorenzo Sorbo for interesting discussions. N.K. is grateful to Galileo Galilei Institute, Florence, Italy, for kind hospitality in the course of this work. This work was supported in

part by the DOE Grant DE-FG03-91ER40674, in part by the NSF Grant PHY-0332258 and in part by a Research Innovation Award from the Research Corporation. 


\section{References}

[1] S. Weinberg, Rev. Mod. Phys. 61, 1 (1989).

[2] A. G. Riess et al., Astron. J. 116, 1009 (1998); S. Perlmutter et al., Astrophys. J. 517, 565 (1999); J. L. Tonry et al., Astrophys. J. 594, 1 (2003); R. A. Knop et al., Astrophys. J. 598, 102 (2003); A. G. Riess et al., Astrophys. J. 607, 665 (2004).

[3] R. Bousso and J. Polchinski, JHEP 0006, 006 (2000); S. Kachru, R. Kallosh, A. Linde and S. P. Trivedi, Phys. Rev. D 68, 046005 (2003).

[4] A. D. Linde, Rept. Prog. Phys. 47, 925 (1984).

[5] S. Weinberg, Phys. Rev. Lett. 59, 2607 (1987).

[6] A. Vilenkin, Phys. Rev. Lett. 74, 846 (1995).

[7] J. L. Feng, J. March-Russell, S. Sethi and F. Wilczek, Nucl. Phys. B 602, 307 (2001).

[8] L. Susskind, arXiv:hep-th/0302219.

[9] T. Banks, M. Dine and E. Gorbatov, JHEP 0408, 058 (2004).

[10] V. A. Rubakov and M. E. Shaposhnikov, Phys. Lett. B 125, 139 (1983).

[11] B. Holdom, ITP-744-STANFORD preprint, 1983.

[12] N. Arkani-Hamed, S. Dimopoulos, N. Kaloper and R. Sundrum, Phys. Lett. B 480, 193 (2000); S. Kachru, M. B. Schulz and E. Silverstein, Phys. Rev. D 62, 045021 (2000).

[13] S. Forste, Z. Lalak, S. Lavignac and H. P. Nilles, Phys. Lett. B 481, 360 (2000).

[14] J. Polchinski and M. J. Strassler, arXiv:hep-th/0003136.

[15] G. R. Dvali, G. Gabadadze and M. Porrati, Phys. Lett. B 485, 208 (2000); G. R. Dvali and G. Gabadadze, Phys. Rev. D 63, 065007 (2001).

[16] N. Kaloper, Phys. Rev. Lett. 94, 181601 (2005) [Erratum-ibid. 95, 059901 (2005)]; Phys. Rev. D 71, 086003 (2005) [Erratum-ibid: D71 (2005) 086003].

[17] Y. Iwasaki, Phys. Rev. D 2, 2255 (1970); H. van Dam and M. J. G. Veltman, Nucl. Phys. B 22, 397 (1970); V. I. Zakharov, JETP Lett. 12 312, (1970).

[18] A. I. Vainshtein, Phys. Lett. B 39 393, (1972).

[19] C. Deffayet, G. R. Dvali, G. Gabadadze and A. I. Vainshtein, Phys. Rev. D 65044026 , (2002).

[20] N. Arkani-Hamed, H. Georgi and M. D. Schwartz, Annals Phys. 305 96, (2003).

[21] V. A. Rubakov, arXiv:hep-th/0303125. 
[22] M. A. Luty, M. Porrati and R. Rattazzi, JHEP 0309, 029 (2003).

[23] A. Nicolis and R. Rattazzi, JHEP 0406, 059 (2004).

[24] K. Koyama, Phys. Rev. D 72, 123511 (2005); D. Gorbunov, K. Koyama and S. Sibiryakov, Phys. Rev. D 73, 044016 (2006).

[25] C. Charmousis, R. Gregory, N. Kaloper and A. Padilla, JHEP 0610, 066 (2006).

[26] C. Deffayet, G. Gabadadze and A. Iglesias, JCAP 0608, 012 (2006).

[27] K. Izumi, K. Koyama and T. Tanaka, arXiv:hep-th/0610282.

[28] M. Carena, J. Lykken, M. Park and J. Santiago, Phys. Rev. D 75, 026009 (2007).

[29] G. Dvali, G. Gabadadze, X. r. Hou and E. Sefusatti, Phys. Rev. D 67, 044019 (2003).

[30] S. L. Dubovsky and V. A. Rubakov, Phys. Rev. D 67, 104014 (2003).

[31] M. Kolanovic, M. Porrati and J. W. Rombouts, Phys. Rev. D 68, 064018 (2003).

[32] G. Gabadadze and M. Shifman, Phys. Rev. D 69, 124032 (2004).

[33] R. Sundrum, Phys. Rev. D 59, 085010 (1999).

[34] H. P. Nilles, A. Papazoglou and G. Tasinato, Nucl. Phys. B 677, 405 (2004); M. L. Graesser, J. E. Kile and P. Wang, Phys. Rev. D 70, 024008 (2004); J. Garriga and M. Porrati, JHEP 0408, 028 (2004); M. Redi, Phys. Rev. D 71, 044006 (2005).

[35] A. Chodos and E. Poppitz, Phys. Lett. B 471, 119 (1999); E. Ponton and E. Poppitz, JHEP 0102, 042 (2001).

[36] A. G. Cohen and D. B. Kaplan, Phys. Lett. B 470, 52 (1999).

[37] R. Gregory, Phys. Rev. Lett. 84, 2564 (2000); JHEP 0306, 041 (2003).

[38] J. W. Chen, M. A. Luty and E. Ponton, JHEP 0009, 012 (2000).

[39] T. Gherghetta and M. E. Shaposhnikov, Phys. Rev. Lett. 85, 240 (2000); T. Gherghetta, E. Roessl and M. E. Shaposhnikov, Phys. Lett. B 491, 353 (2000).

[40] N. Arkani-Hamed, S. Dimopoulos, G. R. Dvali and N. Kaloper, Phys. Rev. Lett. 84, $586(2000)$.

[41] N. Kaloper, JHEP 0405, 061 (2004); AIP Conf. Proc. 743, 318 (2005).

[42] P. Kanti, R. Madden and K. A. Olive, Phys. Rev. D 64, 044021 (2001).

[43] Y. Aghababaie et al., JHEP 0309, 037 (2003); C. P. Burgess, J. Matias and F. Quevedo, Nucl. Phys. B 706, 71 (2005). 
[44] J. M. Cline, J. Descheneau, M. Giovannini and J. Vinet, JHEP 0306, 048 (2003).

[45] O. Corradini, A. Iglesias, Z. Kakushadze and P. Langfelder, Phys. Lett. B 521, 96 (2001); P. Bostock, R. Gregory, I. Navarro and J. Santiago, Phys. Rev. Lett. 92, 221601 (2004).

[46] J. Vinet and J. M. Cline, Phys. Rev. D 70, 083514 (2004); Phys. Rev. D 71, 064011 (2005).

[47] C. de Rham and A. J. Tolley, JCAP 0602, 003 (2006).

[48] A. J. Tolley, C. P. Burgess, D. Hoover and Y. Aghababaie, JHEP 0603, 091 (2006)

[49] H. M. Lee and G. Tasinato, JCAP 0404, 009 (2004); I. Navarro and J. Santiago, JHEP 0502, 007 (2005); E. Papantonopoulos and A. Papazoglou, JCAP 0507, 004 (2005); C. Charmousis and R. Zegers, JHEP 0508, 075 (2005); Phys. Rev. D 72, 064005 (2005); G. Kofinas, Phys. Lett. B 633, 141 (2006).

[50] N. Kaloper and D. Kiley, JHEP 0603, 077 (2006).

[51] M. Peloso, L. Sorbo and G. Tasinato, Phys. Rev. D 73, 104025 (2006).

[52] E. Papantonopoulos, A. Papazoglou and V. Zamarias, arXiv:hep-th/0611311; T. Kobayashi and M. Minamitsuji, arXiv:hep-th/0703029.

[53] J. Scherk and J. H. Schwarz, Phys. Lett. B 82, 60 (1979); Nucl. Phys. B 153, 61 (1979).

[54] J. R. I. Gott, Astrophys. J. 288 422, (1985); P. Laguna and D. Garfinkle, Phys. Rev. D 40, 1011 (1989); B. Linet, Class. Quant. Grav. 7, L75 (1990); M. E. Ortiz, Phys. Rev. D 43, 2521 (1991); I. Cho, Phys. Rev. D 58, 103509 (1998).

[55] M. Gell-Mann and B. Zwiebach, Phys. Lett. B 147, 111 (1984); Nucl. Phys. B 260, 569 (1985).

[56] G. Dvali, G. Gabadadze, O. Pujolas and R. Rahman, arXiv:hep-th/0612016.

[57] P. C. Aichelburg and R. U. Sexl, Gen. Rel. Grav. 2, 303 (1971).

[58] V. Ferrari, P. Pendenza and G. Veneziano, Gen. Rel. Grav. 20, 1185 (1988); H. de Vega and N. Sanchez, Nucl. Ph. B 317, 706 (1989).

[59] N. Kaloper, arXiv:hep-th/0702206.

[60] E. Kiritsis, N. Tetradis and T. N. Tomaras, JHEP 0108, 012 (2001).

[61] S. Corley, D. A. Lowe and S. Ramgoolam, JHEP 0107, 030 (2001).

[62] I. Antoniadis, R. Minasian and P. Vanhove, Nucl. Phys. B 648, 69 (2003).

[63] N. Kaloper and L. Sorbo, JHEP 0508, 070 (2005). 
[64] S. Weinberg, Gravitation and cosmology, J. Wiley and Sons, 1972 (see sec. 13.5 for the discussion of maximally symmetric subspaces, and sec. 9.9 for PPN limit in BransDicke).

[65] T. Dray and G. 't Hooft, Nucl. Phys. B 253, 173 (1985); Class. Quant. Grav. 3, 825 (1986).

[66] K. Sfetsos, Nucl. Phys. B 436, 721 (1995).

[67] J. Garriga and T. Tanaka, Phys. Rev. Lett. 84, 2778 (2000).

[68] T. Tanaka, Phys. Rev. D 69, 024001 (2004).

[69] G. Cacciapaglia, C. Csaki, G. Marandella and J. Terning, JHEP 0702, 036 (2007). 